Illinois Journal of Mathematics

Volume 50, Number 2, Summer 2006, Pages 269-312

S $0019-2082$

\title{
ENTRANCE LAW, EXIT SYSTEM AND LÉVY SYSTEM OF TIME CHANGED PROCESSES
}

\author{
ZHEN-QING CHEN, MASATOSHI FUKUSHIMA, AND JIANGANG YING
}

\begin{abstract}
Let $(X, \widehat{X})$ be a pair of Borel standard processes on a Lusin space $E$ that are in weak duality with respect to some $\sigma$-finite measure $m$ that has full support on $E$. Let $F$ be a finely closed subset of $E$. In this paper, we obtain the characterization of a Lévy system of the time changed process of $X$ by a positive continuous additive functional (PCAF in abbreviation) of $X$ having support $F$, under the assumption that every $m$-semipolar set of $X$ is $m$-polar for $X$. The characterization of the Lévy system is in terms of Feller measures, which are intrinsic quantities for the part process of $X$ killed upon leaving $E \backslash F$. Along the way, various relations between the entrance law, exit system, Feller measures and the distribution of the starting and ending point of excursions of $X$ away from $F$ are studied. We also show that the time changed process of $X$ is a special standard process having a weak dual and that the $\mu$-semipolar set of $Y$ is $\mu$-polar for $Y$, where $\mu$ is the Revuz measure for the PCAF used in the time change.
\end{abstract}

\section{Introduction}

Given a Markov process $X$ on a state space $E$ and a subset $F$ of $E$, we may associate the minimal process $X^{0}$ on $E_{0}=E \backslash F$ and the time changed process $Y$ on $F ; X^{0}$ and $Y$ are obtained from $X$ by killing upon leaving $E_{0}$, and with the time substitution by the inverse of the local time on $F$, respectively. There is yet another associated process that has attracted the interest of researchers for many years: the excursions of $X$ away from the set $F$.

Intuitively, the joint distribution of the starting and ending points of excursions should contribute to the jumping measure of $Y$, as has been verified by explicit computations for the reflecting Brownian motion on a smooth Euclidean domain by P. Hsu [25]. In $\S 5$ of this paper, we shall show that this

Received March 8, 2005; received in final form September 19, 2005.

2000 Mathematics Subject Classification. Primary 60J45, 60J50, 31C25.

The research of the first author is supported in part by NSF Grant DMS-0303310. The research of the second author is supported in part by Grant-in-Aid for Scientific Research of MEXT No. 15540142. The research of the third author is supported in part by NSFC No. 10271109. 
is indeed the case in great generality. One can also naturally guess that the entrance law governing the excursions ought to be determined uniquely by the dual of the minimal process $X^{0}$. When $F$ is just a one point set, this was recently confirmed by M. Fukushima and H. Tanaka [18] for a symmetric diffusion $X$ and by P. J. Fitzsimmons and R. K. Getoor [13] for a general Markov process $X$. In $\S 3$ of the present paper, we shall establish this identification when $F$ is a general finely closed set for a Markov process $X$ possessing its dual.

The stated results in $\S 3$ and $\S 5$ of the present paper will lead us in $\S 5$ to the characterization of the jumping and killing measures of $Y$ by means of the Feller measures, which are the intrinsic quantities for $X^{0}$ and its dual process. The Feller measure was introduced by W. Feller [6] in his study of boundary theory for Markov chains. Such a characterization has been obtained previously by Y. LeJan [30] for a Hunt process associated with a nonsymmetric Dirichlet form under some restrictive condition and quite recently by our joint paper [4] for the most general symmetric Markov processes. The following is a more detailed introduction of the present paper.

Let $X=\left\{X_{t}, \mathbf{P}_{x}\right\}$ be a standard process on a Lusin space $E$ that has a weak dual standard process $\widehat{X}=\left\{\widehat{X}_{t}, \widehat{\mathbf{P}}_{x}\right\}$ with respect to a $\sigma$-finite measure $m$ having full support on $E$. We assume for $X$ that

(A.1) every $m$-semipolar set is $m$-polar.

Fix a subset $F$ of $E$ satisfying

(A.2) $F$ is q.e. finely closed,

(A.3) $\mathbf{P}_{x}\left(\sigma_{F}<\infty\right)>0$ for $m_{0}$-a.e. $x \in E_{0}$, where $E_{0}:=E \backslash F, m_{0}:=\left.m\right|_{E_{0}}$ and $\sigma_{F}:=\inf \left\{t>0: X_{t} \in F\right\}$, the hitting time of $F$ by $X$.

There are two important stochastic objects relevant to the set $F$ : Maisonneuve's exit system for the homogeneous random set

$$
M(\omega):=\left\{t \in[0, \zeta(\omega)): X_{t}(\omega) \in F \text { or } X_{t-}(\omega) \in F\right\},
$$

and the trace process $Y$ on $F$ obtained from $X$ by a time change with respect to a positive continuous additive functional (PCAF in abbreviation) having support $F$. The aim of the present paper is to describe some basic characteristics in these two objects in terms of the specific quantities related to the minimal processes $X^{0}$ and $\widehat{X}^{0}$, which are the subprocesses of $X$ and $\widehat{X}$, respectively, killed upon leaving $E_{0}$.

By the specific quantities, we mean an $X^{0}$-entrance law $\left\{\mu_{t}^{f}, t \geq 0\right\}$ on $E_{0}$ that is characterized by

$$
\widehat{\mathbf{H}} f \cdot m_{0}=\int_{0}^{\infty} \mu_{t}^{f} d t \quad \text { for every } f \in \mathcal{B}^{+}(F)
$$


the Feller measure $U$ and the supplementary Feller measure $V$ that are defined by

$U(f, g):=L^{(0)}\left(\widehat{\mathbf{H}} f \cdot m_{0}, \mathbf{H} g\right), \quad V(f):=L^{(0)}\left(\widehat{\mathbf{H}} f \cdot m_{0}, 1-\mathbf{H} 1\right), \quad f, g \in \mathcal{B}^{+}(F)$.

Here for $x \in E_{0}$,

$$
\mathbf{H} f(x):=\mathbf{E}_{x}\left[f\left(X_{\sigma_{F}}\right) ; \sigma_{F}<\infty\right] \quad \text { and } \quad \widehat{\mathbf{H}} f(x):=\widehat{\mathbf{E}}_{x}\left[f\left(\widehat{X}_{\sigma_{F}}\right) ; \sigma_{F}<\infty\right] \text {, }
$$

and $L^{(0)}$ is the $X^{0}$-energy functional of an excessive measure and an excessive function for $X^{0}$. We emphasize that those quantities are well computable in many examples. The entrance law $\left\{\mu_{t}^{f}, t \geq 0\right\}$ can be expressed in terms of the joint distribution of the hitting time and hitting place of $F$ of the dual process $\widehat{X}$ (see (3.7) below). When $X$ is the $d$-dimensional Brownian motion and $F$ is a compact smooth hypersurface, concrete expressions of the Feller measures $U$ and $V$ are derived in [16, Example 2.1] and [4, Example 2.12], respectively.

The exit system $\left(\mathbf{P}_{x}^{*}, K+J\right)$ defined in $\S 3$ for the set $M(\omega)$ will describe the behaviors of the sample path $X_{t}(\omega)$ for $t$ belonging to the time set $[0, \zeta(\omega)) \backslash$ $M(\omega)$, which is a disjoint union of excursion intervals away from the set $F$. In particular, $\mathbf{P}_{x}^{*}$ for $x \in F$ may be considered as a $\sigma$-finite measure on the space of paths continuously entering from $x$ so that

$$
Q_{t}^{*}(x, B)=\mathbf{E}_{x}^{*}\left[1_{B}\left(X_{t}\right) ; t<\sigma_{F}\right]
$$

is an $X^{0}$-entrance law governing the excursions. In Theorem 3.3, we shall establish an identity linking $Q_{t}^{*}(\cdot, B), B \in \mathcal{B}\left(E_{0}\right)$, to the above mentioned $X^{0}$-entrance law $\mu_{t}^{f}(B)$.

Theorem 3.3 can be regarded as an extension of a part of a recent paper [18], where $F$ is a one-point set. P. Fitzsimmons and R. Getoor [13] has shown a similar formula to Theorem 3.3 for a general right process $X$ and for any excessive measure $m$ of it with $F$ being a one-point set.

Theorem 3.3, which relates the $X^{0}$-entrance law $\mu_{t}^{f}$ to the exit system, is a key of the present paper in the sense that all subsequent theorems will be deduced from it. In Theorem 3.4, the Feller measures $U$ and $V$ are represented as joint distributions of the starting and ending points of excursions in terms of the exit system. In Theorem 4.1, the Feller-Neveu measure $\Theta^{f, g}(d u)$ is represented by a joint distribution of the starting point, ending point and the length of excursions. The Feller measure and the Feller-Neveu measure were first introduced by W. Feller [6] and J. Neveu [34], respectively, for a Markov process on a denumerable state space with a finite number of ideal boundary points. When $F$ is just a one point set, $\Theta(d u)$ is nothing but the Lévy measure of the inverse local time at the point (see [18, Theorem 2.2] and [13]).

As a consequence of Theorem 3.4, the Feller measures are identified in Corollary 3.5 with (generalized) Revuz measures of certain homogeneous random measures involving the starting and ending points of excursions. On the 
other hand, the latter quantities will be identified in Theorem 5.5 of $\S 5$ with the jumping and killing measures $\check{J}, \check{\kappa}$ of the time changed process $Y$ on $F$ obtained from $X$ by a PCAF having support $F$. Combining Corollary 3.5 with Theorem 5.5, we shall get in Theorem 5.6 the following identifications

$$
\check{J}=U+\left.J\right|_{F \times F} \quad \text { and } \quad \check{\kappa}=V+\left.\kappa\right|_{F},
$$

where $J$ and $\kappa$ are the jumping measure and killing measure of $X$, respectively. To be more precise, we shall consider in $\S 5$ the totality $S_{F}$ of smooth measures whose quasi support coincide with $F$ q.e.. We take an arbitrary but fixed $\mu \in S_{F}$ and consider the PCAF $A$ having Revuz measure $\mu$. Then the time changed process $Y$ of $X$ by the inverse of $A$ can be seen to be a right process on $F$ possessing a weak dual right process with respect to $\mu$ and still satisfying condition (A.1). Therefore we can verify that both $X$ and $Y$ are special standard processes and admit their Lévy systems (under the original topologies) for quasi every starting points. The above mentioned jumping and killing measures of them are well defined in terms of their Lévy systems. In particular, the identifications in Theorem 5.6 holds independently of the choice of $\mu \in S_{F}$.

Theorem 5.6 is a generalization of the corresponding part of $\S 2$ of our recent joint paper [4], where a general irreducible $m$-symmetric Markov process $X$ on a Lusin space $E$ and a quasi closed subset $F$ of $E$ with positive capacity are considered. The method employed in [4] is to identify the jumping and killing measure appearing in the Beurling-Deny representation of the quasiregular Dirichlet form on $L^{2}(F ; \mu)$ of the time changed process $Y$ by making full use of the stochastic calculus on martingale additive functionals of $X$ in the Dirichlet form setting.

Under some extra condition, LeJan has obtained in $\S 3$ of [30] the same results as $\S 2$ of [4] for a Hunt process $X$ associated with a non-symmetric sectorial regular Dirichlet form and for a closed set $F$. Along with [29], nice potential theoretic methods were systematically utilized in [30] under the condition that the Dirichlet space is continuously embedded into $L^{2}(E ; m)$. This condition however excludes many interesting examples such as the reflecting Brownian motion on the unit disk while $F$ is the unit circle. Our Theorem 5.6 also extends the corresponding part of $\S 3$ of [30] because the Hunt process associated with a non-symmetric sectorial Dirichlet form is known to satisfy the present condition (A.1) (cf. [29], [38], [9]).

When $X$ is an $m$-symmetric conservative diffusion and $F$ is a closed set, Corollary 3.5 can be readily obtained by a direct computation, as was done in $\S 3$ of the paper by M. Fukushima, P. He and J. Ying [16]. The proof of Theorem 5.5 will be carried out by extending a time change argument in the proof of [16, Theorem 5.1]. In this sense, the present paper can be viewed as an extension of [16] and of a part of [18] methodologically. 
Here we mention a close relevance of the present work to an article of $\mathrm{M}$. Motoo [33], where the Lévy system $(\check{N}, \check{H})$ of the time changed process $Y$ on a closed set $F$ of a Hunt process $X$ was studied under some strong analytic conditions on the resolvent of $X^{0}$. The excursions of $X$ away from $F$ were studied using jump times of the inverse local time and also a variant $\Psi$ of the Feller kernel on $F$ was introduced. It was then shown in [33] that

$$
\int_{0}^{t} \check{N} f\left(Y_{s}\right) d \check{H}_{s} \geq \int_{0}^{t} \Psi f\left(Y_{s}\right) d s, \quad t>0, \quad f \in \mathcal{B}^{+}(F)
$$

with the equality holding if and only if $X$ admits no jump from $F$ to $F$. See M. Blumenthal [1] for an interpretation of Motoo's results by making use of an exit system.

There are many papers on the subject of excursions and exit systems of Markov processes; see [8], [21], [23], [25], [26], [27], [31], to name a few. For example, [23], [8] and [27] studied the excursion laws using the exit system, under the strong duality assumption (which assumes the existence of transition density functions) or the classical duality assumption (which assumes the existence of potential kernels). In contrast, in the present paper we define Feller measures independently of the exit system, and then relate these measures to the joint distributions of the starting and ending points of the excursions defined via exit system.

In the next section, we collect some basic facts for standard processes in weak duality from articles [15], [24], [12], [36] and develop them in a convenient way for later uses. Subsections $\S 2.2, \S 2.3$ and $\S 2.4$ contain some new results proven under the condition (A.1). In $\S 2.2$, we show that the above mentioned homogeneous random set $M(\omega)$ is actually a closed subset of $[0, \infty)$. It will be shown in $\S 2.3$ that the quasi support of a smooth measure coincides with the support of the associated PCAF, extending the corresponding part of [17] for $m$-symmetric Markov processes. Subsection $\S 2.4$ deals with the existence of the associated Lévy system under the original topology.

Our study originated in the work of J. L. Doob [5], where the Douglas integral representation of the Dirichlet integral of a harmonic function on a unit disk was generalized to a general Green space with Martin boundary using Naim's kernel. In subsequent works by M. Fukushima [14] and H. Kunita [28], Naim's kernel was replaced by a Feller kernel (a density function of a Feller measure). In these works, what was given a priori is a minimal process $X^{0}$, and what was searched for was its possible extensions together with their intrinsic boundaries. In a series of papers [16], [4] and the present one, we assume instead that a process on $\mathrm{E}$ and a subset $F$ of $E$ are given in advance and the behaviors of $X$ around $F$ are investigated in relation to Feller measures defined by the absorbed process $X^{0}$ and its dual process. 
Acknowledgments. We are grateful to Pat Fitzsimmons and John Walsh for helpful discussions on the subject of fine topology of Markov processes in weak duality and for helpful comments on a preliminary version of this paper. We also thank the anonymous referee for helpful comments, especially the one concerning the justification of (2.17).

\section{Markov processes in weak duality}

Let $E$ be a Lusin space (i.e., a space that is homeomorphic to a Borel subset of a compact metric space) and $\mathcal{B}(E)$ be the Borel $\sigma$-algebra on $E$. Let $X=\left(\Omega, \mathcal{M}, \mathcal{M}_{t}, X_{t}, \mathbf{P}_{x}, x \in E\right)$ be a standard process on $E$. Here, a standard process on the Lusin space $E$ is a normal, right continuous strong Markov process which is quasi-left continuous on $(0, \zeta)$, where $\zeta$ is the lifetime of the process.

The shift operators $\left\{\theta_{t}, t \geq 0\right\}$ satisfy $X_{s} \circ \theta_{t}=X_{s+t}$ identically for $s, t \geq 0$. Adjoined to the state space $E$ is an extra point $\partial \notin E$; the process $X$ retires to $\partial$ at its "lifetime"

$$
\zeta:=\inf \left\{t \geq 0: X_{t}=\partial\right\}
$$

Denote $E \cup\{\partial\}$ by $E_{\partial}$. The transition semigroup $\left\{P_{t}, t \geq 0\right\}$ of the process $X$ is defined by

$$
P_{t} f(x):=\mathbf{E}_{x}\left[f\left(X_{t}\right)\right]=\mathbf{E}_{x}\left[f\left(X_{t}\right) ; t<\zeta\right] .
$$

(Here and in the sequel, unless mentioned otherwise, we use the convention that a function defined on $E$ takes the value 0 at the cemetery point $\partial$.)

A standard process is said to be Borel if $P_{t} f$ is Borel measurable for each bounded Borel function $f$. The family of all nearly Borel subsets of $E$ with respect to the process $X$ will be denoted by $\mathcal{B}^{n}(E)$. We shall start with a Borel standard process $X$. But a right process or a standard process obtained from $X$ by some transformations such as restriction to an $X$-invariant set, a killing, a time change may no longer be Borel; however these processes have the following weaker measurability:

(2.1) $P_{t}$ maps bounded nearly Borel functions into nearly Borel functions.

Let $m$ be a $\sigma$-finite measure on $(E, \mathcal{B}(E))$ with $\operatorname{supp}[m]=E$. Throughout this paper except for $\S 2.1$ (vi), (vii) and $\S 2.4$, we assume that $X$ is a Borel standard process and there is another Borel standard process

$$
\widehat{X}=\left(\Omega, \widehat{\mathcal{M}}, \widehat{\mathcal{M}}_{t}, \widehat{X}_{t}, \widehat{\mathbf{P}}_{x}, x \in E\right)
$$

that is in weak duality with $X$ with respect to the measure $m$ in the sense that

$$
\int_{E} g(x) \widehat{P}_{t} f(x) m(d x)=\int_{E} f(x) P_{t} g(x) m(d x), \quad f, g \in \mathcal{B}^{+}(E) .
$$


Here $\widehat{P}_{t}$ is the transition semigroup of $\widehat{X}$. For $\alpha \geq 0$, the $\alpha$-resolvents of $X$ and $\widehat{X}$ will be denoted by $G_{\alpha}$ and $\widehat{G}_{\alpha}$, respectively. The point $\partial$ will play the role of the cemetery for $X$ and $\widehat{X}$. The quantities relative to $\widehat{X}$ will be denoted with a hat ${ }^{\wedge}$ and designated by the prefix co-.

We know (cf. [2, (I.9.15)]) that almost surely the left limit of $X_{t}$ exists in $E$ for $t<\zeta$. So without loss of generality, we assume that $X_{t}(\omega)$ has left limits in $E$ for every $t \in(0, \zeta(\omega))$ for each $\omega \in \Omega$.

Under the weak duality assumption, the measure $m$ is an excessive measure of $X$, that is, $m$ is a $\sigma$-finite Borel measure on $\mathcal{B}(E)$ such that $m P_{t} \leq m$ for all $t>0$. Here $m P_{t}$ denotes the measure $\mu$ defined by $\int_{E} f(x) \mu(d x)=$ $\int_{E} P_{t} f(x) m(d x)$ for any Borel function $f \geq 0$ on $E$. Since $X$ is a standard process, we have $\lim _{t \rightarrow 0} m P_{t}=m$ setwise.

2.1. Exceptional sets and fine topology. In this subsection, we list some known basic statements about exceptional sets and fine topology related to $X$ and $\widehat{X}$ which have been presented in $[15, \S 2]$ and in $[24, \S 6]$.

The hitting time of $B \subset E_{\partial}$ is defined by $\sigma_{B}=\inf \left\{t>0, X_{t} \in B\right\}$ with the convention that $\inf \emptyset=\infty$.

A subset $B \subset E$ is said to be $m$-polar if there exists a nearly Borel set $\widetilde{B}$ containing $B$ such that

$$
\mathbf{P}_{m}\left(\sigma_{\widetilde{B}}<\infty\right)=0 .
$$

The set $\widetilde{B}$ above can in fact be chosen to be a Borel subset of $E$. It is known that any $m$-polar set is $m$-negligible. In the sequel, a statement is said to hold quasi-everywhere (q.e. in abbreviation) if it holds except on an $m$-polar set.

For two subsets $B_{1}, B_{2}$ of $E$, we write $B_{1} \subset B_{2}$ q.e. if $B_{1} \backslash B_{2}$ is $m$ polar. Thus $B_{1}=B_{2}$ q.e. if their symmetric difference is $m$-polar, and in this case we call them q.e. equivalent. A subset of $E$ is called q.e. finely open (respectively, q.e. finely closed) if it is q.e. equivalent to a nearly Borel finely open (respectively, closed) set. A subset of $E$ is called m-semipolar if it is q.e. equivalent to a semipolar set. An $m$-semipolar set is therefore a union of a semipolar set and an $m$-polar set.

A function $u$ defined q.e. on $E$ is called finely continuous q.e. if there exists an $m$-polar set $N \in \mathcal{B}^{n}$ such that $E \backslash N$ is finely open and $u$ is nearly Borel measurable and finely continuous on $E \backslash N$.

For $F \in \mathcal{B}^{n}$, we put for $f \in \mathcal{B}^{+}(E), x \in E$,

$$
\begin{gathered}
P_{t}^{0} f(x)=\mathbf{E}_{x}\left[f\left(X_{t}\right) ; t<\sigma_{F}\right] \quad \text { for } t>0, \\
\mathbf{H} f(x)=\mathbf{E}_{x}\left[f\left(X_{\sigma_{F}}\right) ; \sigma_{F}<\infty\right], \quad \mathbf{H}_{\alpha} f(x)=\mathbf{E}_{x}\left[e^{-\alpha \sigma_{F}} f\left(X_{\sigma_{F}}\right)\right], \quad \alpha>0 .
\end{gathered}
$$

In the following, we will use $(u, v)$ to denote the inner product of $u, v$ in $L^{2}(E, m)$, that is, $(u, v):=\int_{E} u(x) v(x) m(d x)$. For a set $A$, we use $1_{A}$ to 
denote its indicator function, that is,

$$
1_{A}(x):= \begin{cases}1 & \text { if } x \in A, \\ 0 & \text { if } x \notin A .\end{cases}
$$

The following facts are known.

(i) When $F$ is Borel, the following hold for $f, g \in \mathcal{B}^{+}(E)$ :

$$
\begin{gathered}
\left(\widehat{P}_{t}^{0} f, g\right)=\left(f, P_{t}^{0} g\right) \quad \text { for } t>0, \\
\left(\widehat{\mathbf{H}}_{\alpha} \widehat{G}_{\alpha} f, g\right)=\left(f, \mathbf{H}_{\alpha} G_{\alpha} g\right) \quad \text { for every } \alpha>0 .
\end{gathered}
$$

(ii) $m$-polarity and $m$-co-polarity are equivalent.

Clearly the identities (2.3) and (2.4) extend to $F \in \mathcal{B}^{n}$. We note here that, although $F \in \mathcal{B}^{n}$ is not necessarily co-nearly Borel measurable, there are Borel sets $B_{1}, B_{2}$ such that $B_{1} \subset F \subset B_{2}$ and $B_{2} \backslash B_{1}$ is $m$-polar and hence $m$-co-polar, so that the left hand sides of the above identities make sense.

(iii) If $u$ is finely continuous q.e. on $E$ and $u \geq 0 \mathrm{~m}$-a.e. on a finely open set $G \in \mathcal{B}^{n}$, then $u \geq 0$ q.e. on $G$.

A set $E_{1} \in \mathcal{B}^{n}$ is called $X$-invariant if for every $x \in E_{1}$

$\mathbf{P}_{x}\left(X_{t} \in E_{1}\right.$ for every $t \in[0, \zeta)$ and $X_{t-} \in E_{1}$ for every $\left.t \in(0, \zeta)\right)=1$.

The restriction of $X$ to an $X$-invariant set $E_{1}$ is a standard process on $E_{1}$. We say that a set $N$ is properly exceptional if $N \in \mathcal{B}^{n}, m(N)=0$ and $E \backslash N$ is $X$-invariant. A properly exceptional set is $m$-inessential in the sense of [24] but the converse is not true.

(iv) A set $N$ is $m$-polar if and only if $N$ is contained in a properly exceptional set $\tilde{N}$. The set $\tilde{N}$ can be taken to be Borel.

A function $u$ is q.e. finely continuous on $E$ if and only if there exists a Borel properly exceptional set $N$ such that $u$ is Borel measurable and finely continuous on $E \backslash N$.

The assertions (i), (ii) and (iii) were proved in [15]. The first assertion of (iv) was proved in $[15]$ and in $[24,(6.12)]$ with $\tilde{N}$ being taken to be an $m$ inessential set, but the proof of [17, Theorem 4.1.1] extends to give the above stronger assertion by making use of [24, (15.7)].

(v) The following two conditions are equivalent:

Every $m$-semipolar set is $m$-polar.

A function is q.e. finely continuous if and only if it is q.e. co-finely continuous. 
In [15], condition (2.6) was proved to be equivalent to the condition that every semipolar set is $m$-polar, which is obviously equivalent to (2.5).

Finally, for later use in $\S 5$ for a time changed process, we add to our list two statements shown in [24] for a pair of right processes $(X, \widehat{X})$ in weak duality with respect to $m$ (the standardness is not needed here).

(vi) For $B \in \mathcal{B}^{n}(E), B$ is $m$-semipolar if and only if

$$
\mathbf{P}_{m}\left(X_{t} \in B \text { for uncountably many } t\right)=0 .
$$

If $B$ is $m$-semipolar, then

$$
\mathbf{P}_{x}\left(X_{t} \in B \text { for uncountably many } t\right)=0 \quad \text { for q.e. } x \in E \text {. }
$$

The second assertion in the above statement is immediate from [2, (II.3.4)].

(vii) $m$-semipolarity and $m$-co-semipolarity are equivalent.

2.2. Closedness of a homogeneous random set. From now on, $X$ and $\widehat{X}$ are two Borel standard processes $X, \widehat{X}$ in weak duality with respect to $m$ with an additional assumption that for the process $X$,

(A.1) every $m$-semipolar set is $m$-polar.

REMARK 2.1. Since $m$-polar is $m$-semipolar and $m$-co-polar is $m$-co-semipolar, by (vii), the assumption (A.1) amounts to saying that $m$-polarity, $m$ semipolarity, $m$-co-polarity and $m$-co-semipolarity are all the same.

In this subsection, we formulate a lemma that is important in the next section. A point $x \in E$ is called regular for a set $F \in \mathcal{B}^{n}$ if $\mathbf{P}_{x}\left(\sigma_{F}=0\right)=1$. The set of all regular points for $F$ is denoted by $F^{r}$.

We consider the random subset $M(\omega)$ of $[0, \zeta)$ defined by

$$
M(\omega):=\left\{t \in[0, \zeta(\omega)): X_{t}(\omega) \in F \text { or } X_{t-}(\omega) \in F\right\}, \quad \omega \in \Omega,
$$

with the convention that $X_{0-}(\omega)=X_{0}(\omega)$.

Lemma 2.2. Let $F \in \mathcal{B}^{n}$ be q.e. finely closed. Then the random set $M(\omega)$ defined by $(2.7)$ is a relatively closed subset of $[0, \zeta(\omega)) \mathbf{P}_{x}$-a.s. for q.e. $x \in E$.

Proof. Since $F^{r} \subset F$ q.e. by assumption and $F \backslash F^{r}$ is semipolar and hence $m$-polar by (A.1), we can choose a properly exceptional set $N$ containing the symmetric difference of $F$ and $F^{r}$ by (vi). We then have for $F_{1}=F \backslash N$

$$
F_{1}^{r}=F_{1} \quad \text { and } \quad \mathbf{P}_{x}\left(\sigma_{F}=\sigma_{F_{1}}\right)=1 \quad \text { for } x \in E \backslash N .
$$

Moreover, for $x \in E \backslash N, \mathbf{P}_{x}$-a.s.,

$$
M(\omega)=M_{1}(\omega):=\left\{t \in[0, \zeta(\omega)): X_{t}(\omega) \in F_{1} \text { or } X_{t-}(\omega) \in F_{1}\right\} .
$$


In view of the right continuity of the 1-excessive function $f_{1}(x)=\mathbf{E}_{x}\left[e^{-\sigma_{F}}\right]$ along the path $X_{t}$, it is clear that the set $M(\omega)$ is righthand closed $\mathbf{P}_{x}$-a.s. for $x \in E \backslash N$.

To show the lefthand closedness, we proceed as follows. Under the weak duality condition, it can be shown using an associated stationary process as in $[24,(9.6)]$ (cf. $[32, \S 6],[40])$ that

$t \mapsto f\left(X_{t-}\right)$ is left-continuous on $(0, \zeta)$ for each q.e. co-finely continuous $f$,

$\mathbf{P}_{m}$-a.s. and consequently $\mathbf{P}_{x}$-a.s. for q.e. $x \in E$ by (iii), (iv) of $\S 2.1$.

Define $f_{n}(x)=\mathbf{E}_{x}\left[e^{-n \sigma_{F}}\right]$ for $x \in E$. Note that $f_{n}$ is $n$-excessive for the process $X$, and hence it is finely continuous. According to the assumption (A.1) and $(\mathrm{v})$ of $\S 2.1, f_{n}$ is q.e. co-finely continuous on $E$. Thus we have for q.e. $x \in E, \mathbf{P}_{x}$-a.s.,

$$
\begin{aligned}
t & \mapsto f_{n}\left(X_{t-}\right) \text { is left-continuous and } \\
t & \mapsto f_{n}\left(X_{t}\right) \text { is right continuous having left limit. }
\end{aligned}
$$

In particular, as $t \mapsto X_{t}$ can have at most countably many discontinuous points, we have for q.e. $x \in E, \mathbf{P}_{x}$-a.s.

$$
\lim _{s \uparrow t} f_{n}\left(X_{s}\right)=\lim _{s \uparrow t} f_{n}\left(X_{s-}\right)=f_{n}\left(X_{t-}\right) \quad \text { for every } t \in(0, \zeta) .
$$

Since $f_{n}(x)$ decreases to $1_{F^{r}}(x)=1_{F}(x)$ as $n \uparrow \infty$ for $x \in E \backslash N$, hence by (2.8) for q.e. $x \in E \backslash N, \mathbf{P}_{x^{-}}$a.s. and for $t<\zeta$,

$$
\begin{aligned}
\limsup _{s \uparrow t} 1_{F}\left(X_{s-}\right) & \leq \lim _{n \rightarrow \infty} \limsup _{s \uparrow t} f_{n}\left(X_{s-}\right) \\
& =\lim _{n \rightarrow \infty} f_{n}\left(X_{t-}\right) \\
& =1_{F}\left(X_{t-}\right)
\end{aligned}
$$

and similarly,

$$
\limsup _{s \uparrow t} 1_{F}\left(X_{s}\right) \leq 1_{F}\left(X_{t-}\right),
$$

for q.e. $x \in E \backslash E$. Hence for q.e. $x \in E, \mathbf{P}_{x}$-a.s., if $t_{n} \in M(\omega)$ and $t_{n} \uparrow t$ with $t<\zeta$, then $X_{t-} \in F$ and so $t \in M(\omega)$. This shows that $M(\omega) \cap[0, \zeta(\omega))$ is a closed subset of $[0, \zeta(\omega))$ and so $M(\omega)$ is closed.

2.3. Smooth measures and positive continuous additive functionals. We continue to consider a pair of Borel standard processes $(X, \widehat{X})$ that are in weak duality with respect to $m$ under the assumption (A.1).

Let $\mu$ be a Borel measure on $E$ charging no $m$-polar set. A set $F \subset E$ is said to be a quasi-support of $\mu$ if the next two conditions are satisfied:

$$
F \text { is q.e. finely closed and } \mu(E \backslash F)=0 \text {. }
$$

$$
\text { If } \tilde{F} \text { is another set with property (2.9), then } F \subset \tilde{F} \text { q.e. }
$$


The quasi support of $\mu$ is unique up to q.e. equivalence. Since a closed set is finely closed, the quasi-support of $\mu$, if it exists, is contained in the topological support of $\mu$ q.e. Analogously to [17, Theorem 4.6.2], we have the following criterion of the quasi support.

We denote by $\mathcal{D}$ the space of all non-negative functions $u$ on $E$ such that $u=f_{1}-f_{2}$ for some bounded 1-excessive functions $f_{1}, f_{2}$ on $E$ with respect to $X$.

Proposition 2.3. The following conditions are equivalent to each other for any Borel measure $\mu$ on $E$ charging no m-polar set and for any q.e. finely closed set $F \subset E$ :

(i) $F$ is a quasi support of $\mu$.

(ii) For every function $u$ in $\mathcal{D}, u=0 \mu$-a.e. on $E$ if and only if $u=0$ q.e. on $F$.

(iii) For any q.e. finely continuous function $u, u=0 \mu$-a.e. on $E$ if and only if $u=0$ q.e. on $F$.

Proof. (i) $\rightarrow$ (iii): Suppose condition (i) holds. Then "if" part of (iii) is trivially true. Let $u$ be a q.e. finely continuous function that vanishes $\mu$-a.e. on $E$. Then, $\widetilde{F}:=\{x \in E: u(x)=0\}$ is q.e finely closed having $\mu(\widetilde{F})=0$ and hence $F \subset \tilde{F}$ q.e.. Therefore $u=0$ q.e. on $F$, proving the "only if" part of (iii).

(iii) $\rightarrow$ (ii): This is true trivially.

(ii) $\rightarrow$ (i): Suppose condition (ii) holds. Define

$\mathcal{N}_{\mu}:=\left\{u \in \mathcal{D}: \int_{E}|u| d \mu=0\right\} \quad$ and $\quad \mathcal{D}_{F^{c}}=\{u \in \mathcal{D}: u=0$ q.e. on $F\}$.

Then condition (ii) can be rephrased as $\mathcal{N}_{\mu}=\mathcal{D}_{F^{c}}$. Assume this holds and define

$$
v_{F}(x):=\mathbf{E}_{x}\left[\int_{0}^{\sigma_{F}} e^{-s_{1}} 1_{E}\left(X_{s}\right) d s\right]=1-\mathbf{E}_{x}\left[e^{-\sigma_{F} \wedge \zeta}\right] \quad \text { for } x \in E .
$$

Note that $v_{F}(x)=G_{1} 1(x)-\mathbf{H}_{1} G_{1} 1(x)$ is in $\mathcal{D}$ and $v_{F}=0$ q.e. on $F$; in other words, $v_{F} \in \mathcal{D}_{F^{c}}$. So $v_{F} \in \mathcal{N}_{\mu}$ and hence $\int_{E} v_{F}(x) \mu(d x)=0$. Since $F^{c}$ is q.e. finely open, by the definition of $v_{F}, v_{F}>0$ q.e. on $F^{c}$. Therefore $\mu(E \backslash F)=0$. Consider another q.e. finely closed set $F_{1}$ with $\mu\left(E \backslash F_{1}\right)=0$. Since $v_{F_{1}}=0$ q.e. on $F_{1}, v_{F_{1}}$ belongs to $\mathcal{N}_{\mu}=\mathcal{D}_{F^{c}}$. Since $v_{F_{1}}>0$ q.e. on $F_{1}^{c}$, it follows that $F \subset F_{1}$ q.e. and hence $F$ is a quasi support of $\mu$, proving (i).

Corollary 2.4. Any q.e. finely closed set F admits a bounded Borel measure charging no m-polar set whose quasi support equals $F$ q.e. 
Proof. Let $F$ be a q.e. finely closed set. Take a strictly positive bounded $m$-integrable function $f$ on $E$ and put

$$
\mu(B)=\int_{E} f(x) \mathbf{H}_{1} 1_{B}(x) m(d x)=\mathbf{E}_{f \cdot m}\left[e^{-\sigma_{F}} 1_{B}\left(X_{\sigma_{F}}\right)\right], \quad B \in \mathcal{B}(E) .
$$

Clearly $\mu$ is a finite measure charging no $m$-polar set with $\mu(E \backslash F)=0$. If $u \in \mathcal{D}$ vanishes $\mu$-a.e., then

$$
\left(f, \mathbf{H}_{1} u\right)=\int_{E} u(y) \mu(d y)=0
$$

and so $\mathbf{H}_{1} u=0 \mathrm{~m}$-a.e. on $E$. Since $\mathbf{H}_{1} u \in \mathcal{B}$ and is finely continuous, $\mathbf{H}_{1} u=0$ q.e. on $E$ by (iii). In particular, $u=\mathbf{H}_{1} u=0$ q.e. on $F$. Hence $\mu$ satisfies condition (ii) of Corollary 2.3.

For a Borel measure charging no $m$-polar set, its co-quasi support is well defined by replacing "q.e. finely closed" in (2.8) by "q.e. co-finely closed".

Corollary 2.5. Let $\mu$ be a Borel measure on $E$ charging no m-polar set. Then the quasi support of $\mu$ and the co-quasi support of $\mu$ are q.e. equivalent.

Proof. This follows from (v) in $§ 2.1$ and Proposition 2.3 (iii).

We call a functional $A=\left\{A_{t}(\omega), t \geq 0, \omega \in \Omega\right\}$, a positive continuous additive functional (PCAF in abbreviation) of $X$ if the following conditions are satisfied:

(1) $A$ is adapted to the minimum augmented admissible filtration $\left\{\mathcal{F}_{t}\right\}_{t \geq 0}$ generated by $X$.

(2) There exist a set $\Lambda \in \mathcal{F}_{\infty}$ and a properly exceptional set $N \subset E$ such that

$$
\mathbf{P}_{x}(\Lambda)=1 \text { for every } x \in E \backslash N, \quad \text { and } \quad \theta_{t} \Lambda \subset \Lambda \text { for } t>0 \text {, }
$$

and, moreover, for each $\omega \in \Omega, t \mapsto A$.( $\omega)$ is non-negative finite continuous on $[0, \zeta(\omega)), A_{0}(\omega)=0, A_{t}(\omega)=A_{\zeta(\omega)}(\omega)$ for every $t \geq$ $\zeta(\omega)$, and for every $t, s>0$,

$$
A_{t+s}(\omega)=A_{s}(\omega)+A_{t}\left(\theta_{s} \omega\right) .
$$

The sets $\Lambda$ and $N$ are called the defining set and the exceptional set of $A$, respectively. A PCAF for which $N=\emptyset$ is called a PCAF in the strict sense. In short, a PCAF is a PCAF in the strict sense of the restricted standard process $\left.X\right|_{E \backslash N}$ for some properly exceptional set $N$. Two PCAF's $A$ and $\widetilde{A}$ are regarded to be equivalent if $\mathbf{P}_{x}\left(A_{t}=\widetilde{A}_{t}\right)=1$ for q.e. $x \in E$ for each $t>0$. The latter is equivalent to

$$
\mathbf{P}_{x}\left(A_{t}=\widetilde{A}_{t} \text { for every } t \geq 0\right)=1 \quad \text { for q.e. } x \in E \text {. }
$$


If $A$ is a PCAF of $X$, then the Revuz measure of $A$ (with respect to the excessive measure $m$ ) is the positive measure $\mu$ defined for $\mathcal{B}(E)$-measurable $f \geq 0$ by the monotone limit

$$
\int_{E} f(x) \mu(d x)=\uparrow \lim _{t \downarrow 0} \frac{1}{t} \mathbf{E}_{m}\left[\int_{0}^{t} f\left(X_{s}\right) d A_{s}\right] .
$$

Here $\uparrow \lim _{t \downarrow 0}$ means the quantity is increasing as $t \downarrow 0$. Obviously the Revuz measure is uniquely determined by the equivalence class of PCAF's and charges no $m$-polar set.

The family of all PCAF's of $X$ is denoted by $\mathbf{A}_{c}^{+}$. For $A \in \mathbf{A}_{c}^{+}$, the associated $\alpha$-potential $U_{A}^{\alpha} f$ is defined for $\alpha \geq 0, f \in \mathcal{B}(E)$, by

$$
U_{A}^{\alpha} f(x)=\mathbf{E}_{x}\left[\int_{0}^{\infty} e^{-\alpha t} f\left(X_{t}\right) d A_{t}\right], \quad x \in E \backslash N,
$$

where $N$ is an exceptional set for $A$. The next fundamental Revuz formula valid under the present weak duality setting (see [24, (9.3)]) will be utilized in $\S 3$ and $\S 5$ :

$$
\left(f, U_{A}^{\alpha} g\right)=\left\langle\widehat{G}_{\alpha} f, g \cdot \mu\right\rangle, \quad f, g \in \mathcal{B}^{+}(E),
$$

where $\mu$ is the Revuz measure of $A$.

The support of $A \in \mathbf{A}_{c}^{+}$is defined by

$$
\check{F}=\left\{x \in E \backslash N: \mathbf{P}_{x}\left(\inf \left\{t>0: A_{t}(\omega)>0\right\}=0\right)=1\right\},
$$

where $N$ is an exceptional set for $A$. The support $\check{F}$ is nearly Borel measurable and finely closed with respect to the process $\left.X\right|_{E \backslash N}$; moreover (cf. [2, p. 215])

$$
A_{t}=\int_{0}^{t} 1_{\breve{F}}\left(X_{s}\right) d A_{s}, \quad \mathbf{P}_{x} \text {-a.s. for } x \in E \backslash N .
$$

The support $\check{F}$ is uniquely determined up to q.e. for equivalent PCAF's.

Proposition 2.6. The support $\check{F}$ of $A \in \mathbf{A}_{c}^{+}$is a quasi support of the Revuz measure $\mu_{A}$ of $A$.

Proof. $\check{F}$ is q.e. finely closed as was noted above and $\mu_{A}(E \backslash \check{F})=0$ in view of (2.11) and (2.15). Therefore it suffices to verify the condition (iii) of Proposition 2.3: if $u$ is q.e. finely continuous and vanishes $\mu_{A}$-a.e. on $E$, then $u=0$ q.e. on $\check{F}$. But this can be shown in exactly the same way as in the proof of [17, Theorem 5.1.5].

When $X=\widehat{X}$ and the associated Dirichlet form on $L^{2}(E ; m)$ is regular, the family (of equivalence classes of) $\mathbf{A}_{c}^{+}$was shown in [17] to be in one to one correspondence with the class of smooth measures under (2.10). This has been extended by Fitzsimmons-Getoor [12] to a general right process with respect to its excessive measure $m$. A Borel measure $\mu$ on $E$ is called smooth 
if it charges no $m$-polar set and there exists an increasing sequence of finely open sets $\left\{E_{n}\right\}$ such that $\mu\left(E_{n}\right)<\infty$ for all $n$ and

$$
\mathbf{P}_{m}\left(\lim _{n \rightarrow \infty} \tau_{E_{n}}<\zeta\right)=0
$$

where $\tau_{E_{n}}:=\inf \left\{t>0: X_{t} \notin E_{n}\right\}$. The class of all smooth measures is denoted by $S$. In [12], the smoothness of a measure $\mu$ is defined as above but with a stronger requirement that $m$ charges no $m$-semipolar set, which, however, is equivalent to the above definition under the present assumption (A.1).

The next proposition is a special case of [12, Theorem 3.11].

Proposition 2.7. The equivalence classes of $\mathbf{A}_{c}^{+}$and $S$ are in one to one correspondence by the Revuz relation (2.11).

By Proposition 2.6 and Proposition 2.7, we conclude that any smooth measure $\mu \in S$ admits a quasi support which equals the support of the corresponding PCAF $A$.

An increasing sequence of q.e. finely open subsets $\left\{E_{n}, n \geq 1\right\}$ is called an $X$-nest if condition (2.16) is satisfied. An $\widehat{X}$-nest can be defined analogously. An $X$-nest $\left\{E_{n}, n \geq 1\right\}$ appearing in the above definition of the smooth measure $\mu$ will be said to be associated with $\mu$.

Proposition 2.8.

(i) $\left\{E_{n}, n \geq 1\right]$ is an $X$-nest if and only if it is an $\widehat{X}$-nest,

(ii) A Borel measure $\mu$ on $E$ is a smooth measure for $X$ if and only if it is a smooth measure for $\widehat{X}$.

(iii) Let $\mu \in S$ and let $A, \widehat{A}$ be the PCAF's of $X, \widehat{X}$, respectively, having Revuz measure $\mu$. Then for every $\alpha>0$, there exists an $X$-nest $\left\{E_{n}\right\}$ associated with $\mu$ such that the $\alpha$-potential and the co- $\alpha$-potential $U_{A_{n}}^{\alpha} 1, \widehat{U}_{\hat{A}_{n}}^{\alpha} 1$ of $A_{n}:=1_{E_{n}} \cdot A, \widehat{A}_{n}:=1_{E_{n}} \cdot \widehat{A}$ are bounded on $E$ for each $n$. Here $\left(1_{E_{n}} \cdot A\right)_{t}:=\int_{0}^{t} 1_{E_{n}}\left(X_{s}\right) d A_{s}$ and $\left(1_{E_{n}} \cdot \widehat{A}\right)_{t}:=\int_{0}^{t} 1_{E_{n}}\left(\widehat{X}_{s}\right) d \widehat{A}_{s}$.

Proof. (i) Under the condition (A.1), it is known that a set $A$ is q.e. finely open for $X$ if and only if it is q.e. finely open for $\widehat{X}$. Take $f$ and $g$, two strictly positive bounded functions on $E$ so that $f, g \in L^{1}(E, m)$. Then $\widehat{G}_{1} f$ and $G_{1} g$ are strictly positive on $E$ q.e.. Let $\left\{E_{n}, n \geq 1\right\}$ be an $X$-nest. Define $F_{n}:=E \backslash E_{n}$. Then by (2.4), we have for every $n \geq 1$

$\int_{E} g(x) \widehat{\mathbf{E}}_{x}\left[e^{-\widehat{\sigma}_{F_{n}}} \widehat{G}_{1} f\left(\widehat{X}_{\widehat{\sigma}_{F_{n}}}\right)\right] m(d x)=\int_{E} f(x) \mathbf{E}_{x}\left[e^{-\sigma_{F_{n}}} G_{1} g\left(X_{\sigma_{F_{n}}}\right)\right] m(d x)$.

It follows from $\left\{E_{n}, n \geq 1\right\}$ being an $X$-nest that

$$
\lim _{n \rightarrow \infty} \int_{E} g(x) \widehat{\mathbf{E}}_{x}\left[e^{-\widehat{\sigma}_{F_{n}}} \widehat{G}_{1} f\left(\widehat{X}_{\widehat{\sigma}_{F_{n}}}\right)\right] m(d x)=0 .
$$


Define $T:=\lim _{n \rightarrow \infty} \widehat{\sigma}_{F_{n}}$. By the quasi-left continuity of $\widehat{X}$ on $[0, \widehat{\zeta})$, we have from the above display and the Fatou lemma by letting $n \rightarrow \infty$ that

$$
\int_{E} g(x) \widehat{\mathbf{E}}_{x}\left[e^{-T} \widehat{G}_{1} f\left(\widehat{X}_{T}\right) ; T<\widehat{\zeta}\right] m(d x)=0 .
$$

It follows that $\mathbf{P}_{m}(T<\widehat{\zeta})=0$ and so $\left\{E_{n}, n \geq 1\right\}$ is an $\widehat{X}$-nest. Interchanging the role of $X$ and $\widehat{X}$, we see that every $\widehat{X}$-nest is an $X$-nest.

(ii) follows immediately from (i) and the definition of smooth measure.

(iii) First assume that $\mu(E)<\infty$. Then by $(2.13), U_{A}^{\alpha} 1(x), \widehat{U}_{\widehat{A}}^{\alpha} 1(x)$ are finite $m$-a.e. and hence q.e. on $E$. Define, for $n \geq 1$,

$$
E_{n}:=\left\{x \in E: U_{A}^{\alpha} 1(x)<n, \widehat{U}_{\hat{A}}^{\alpha} 1(x)<n\right\} .
$$

Clearly, $\left\{E_{n}, n \geq 1\right\}$ are q.e. finely open sets increasing to $E$ q.e. and consequently an $X$-nest. Furthermore, we have for each $n \geq 1$,

$$
U_{A_{n}}^{\alpha} 1(x)=\mathbf{E}_{x}\left[e^{-\alpha \sigma_{E_{n}}} U_{A_{n}}^{\alpha} 1\left(X_{\sigma_{E_{n}}}\right)\right] \leq n \quad \text { for q.e. } x \in E .
$$

Similarly, $\widehat{U}_{\hat{A}_{n}}^{\alpha} 1$ is dominated by $n$.

For a general smooth measure with an associated $X$-nest $\left\{G_{\ell}\right\}$, the measure $\mu_{n}=1_{G_{\ell}} \cdot \mu$ is finite for each $\ell$, and admits an $X$-nest $\left\{E_{n}^{(\ell)}\right\}$ possessing the above property for $A_{\ell}=1_{G_{\ell}} \cdot A, \widehat{A}_{\ell}=1_{G_{\ell}} \cdot \widehat{A}$. Then

$$
E_{n}=\bigcup_{\ell=1}^{n}\left(G_{\ell} \cap E_{n}^{(\ell)}\right), n=1,2, \cdots
$$

is a desired $X$-nest associated with $\mu$.

2.4. Special standard process and Lévy system. We continue to consider a pair of standard processes $X, \widehat{X}$ in weak duality with respect to $m$ under the assumption (A.1). But in this subsection, we do not assume that $X$ and $\widehat{X}$ are Borel and we allow their weaker measurability (2.1). We call $X \mu$-special standard if, for any sequence of stopping times $T_{n} \uparrow T$, $\mathcal{F}_{T}^{\mu}=\sigma\left(\bigcup_{n=1}^{\infty} \mathcal{F}_{T_{n}}^{\mu}\right)$, where $\left\{\mathcal{F}_{t}^{\mu}, t \geq 0\right\}$ denotes the augmented filtration generated by $X$ under $\mathbf{P}_{\mu}$. $X$ is said to be special standard if it is $\mu$-special standard for any initial law $\mu$.

LEMMA 2.9. Under the assumption (A.1), $X$ is $m$-special standard. There exists a properly exceptional set $N$ such that the restricted process $\left.X\right|_{X \backslash N}$ is special standard. The same is true for $\widehat{X}$.

REMARK 2.10. This lemma is a consequence of Theorem 16.19 and Theorem 16.21 and remarks preceding them in [24]. We remark here that this lemma holds more generally for right processes $X$ and $\widehat{X}$ possessing left limits in $E$ up to the lifetimes which are in weak duality with respect to $m$ and the first part of this lemma holds if every $m$-semipolar set is $m$-co-polar while the 
second part of this lemma holds if every $m$-co-semipolar set is $m$-polar. This remark will be applied in $\S 5$ to a time changed processes of $X$ and $\widehat{X}$.

Any right process is known to admit a Lévy system under the Ray topology (see $[36,(73.1)])$. But we like to have a Lévy system under the original topology.

Lemma 2.11. Suppose that $X$ is a special standard process on $E$ with lifetime $\zeta$. For this lemma, we use $X_{t-}^{0}$ and $X_{t-}$ to denote the left limits under the original topology on $E$ and under the Ray topology, respectively. Then for any $x \in E, \mathbf{P}_{x^{-}-a . s .}$ on $\{\zeta<\infty\}, X_{\zeta-}^{0}$ exists in $E$ if and only if $X_{\zeta-} \in E$. In this case, these two limits are the same.

Proof. By [36, Remark (46.3)], we know that if $X_{\zeta-} \in E$ exists in the Ray topology, then $X_{\zeta-}^{0}$ exists in $E$ and coincides with $X_{\zeta-}$.

Note that (see $[36,(47.6)]$ ) under the Ray topology, $X$ is a Hunt process. By [36, Theorem (46.2)], the event $\left\{X_{t-}^{0}\right.$ does not exit in $\left.E\right\}$ is predictably meager. So in particular, we have a.s. on $\left\{X_{\zeta-}^{0}\right.$ does not exit in $\left.E\right\}$ that $X_{\zeta-}=X_{\zeta}=\partial$.

So it remains to show that $\mathbf{P}_{x}(A)=0$ for every $x \in E$, where

$$
A:=\left\{\zeta<\infty \text { and } X_{\zeta-}^{0} \text { exists in } E \text { but differs from } X_{\zeta-}\right\} .
$$

Define

$$
\zeta_{p}:= \begin{cases}\zeta, & \text { if } X_{\zeta-}=\partial \text { and } \zeta<\infty \\ \infty, & \text { otherwise. }\end{cases}
$$

which is predictable by [36, (44.5)]. So there is an increasing sequence of stopping times $\left\{T_{n}, n \geq 1\right\}$ such that $T_{n}<\zeta_{p}$ and $\lim _{n \rightarrow \infty} T_{n}=\zeta_{p}$. According to [36, Theorem (46.2)], the event

$$
\left\{X_{t-}^{0} \text { exists in } E \text { but differs from } X_{t-}\right\}
$$

is also predictably meager. So $A \subset\left\{\zeta_{p}<\infty\right\}$. Define $h(x):=\mathbf{P}_{x}(A)$. Then $h$ is excessive on $E$. Define

$$
M_{t}=\mathbf{E}_{x}\left[1_{A} \mid \mathcal{F}_{t}\right],
$$

which is a bounded martingale. From the Markov property of $X$, we have $M_{t}=h\left(X_{t}\right)$ for $t<\zeta_{p}$. Since $X$ is special standard, the filtration $\left\{\mathcal{F}_{t}, t \geq 0\right\}$ is quasi-left continuous by $[36,(47.6)]$. As $\zeta_{p}$ is predictable, $M_{t}$ is continuous at $t=\zeta_{p}$. We know from $[36,(42.1)]$ that $\bar{P}_{0} h\left(X_{-}\right)$is the predictable projection of $h(X)$, where $\left\{\bar{P}_{t}, t \geq 0\right\}$ denotes the (Ray) semigroup of $X$, while for the martingale $\left\{M_{t}, t \geq 0\right\}$ its predictable projection is $\left\{M_{t-}, t \geq 0\right\}$ with $M_{0-}:=M_{0}$. Since $\bar{P}_{0} h=h$ on $E$ and $X_{\zeta_{p}-}=\partial$, we conclude that

$$
\lim _{n \rightarrow \infty} M_{T_{n}}=\lim _{n \rightarrow \infty} M_{T_{n}-}=\lim _{n \rightarrow \infty} \bar{P}_{0} h\left(X_{T_{n}-}\right)=\bar{P}_{0} h\left(X_{\zeta_{p}-}\right)=0
$$


on $\left\{\zeta_{p}<\infty\right\}$. This implies that

$$
1_{A}=1_{\{\zeta<\infty\}} M_{\zeta}=\lim _{n \rightarrow \infty} M_{T_{n}} 1_{\left\{\zeta_{p}<\infty\right\}}=0 \quad \mathbf{P}_{x^{-}} \text {-a.s. }
$$

for every $x \in E$. This proves the lemma.

By virtue of $[36,(73.1),(47.10)]$ and Lemma 2.11, we can conclude as follows:

LEMma 2.12. Any special standard process $X$ on $E$ has a Lévy system $(N, H)$. That is, $N(x, d y)$ is a kernel on $\left(E_{\partial}, \mathcal{B}\left(E_{\partial}\right)\right)$ and $H$ is a PCAF of $X$ in the strict sense with bounded 1-potential such that for any nonnegative Borel function $f$ on $E \times E_{\partial}$ that vanishes on the diagonal and is extended to be zero elsewhere,

$$
\mathbf{E}_{x}\left[\sum_{s \leq t} f\left(X_{s-}, X_{s}\right)\right]=\mathbf{E}_{x}\left[\int_{0}^{t} \int_{E_{\partial}} f\left(X_{s}, y\right) N\left(X_{s}, d y\right) d H_{s}\right]
$$

for every $x \in E$ and $t \geq 0$, where $X_{\zeta-}$ is defined by

$$
X_{\zeta-}:= \begin{cases}\lim _{t \uparrow \zeta} X_{t}, & \text { if the limit } \lim _{t \uparrow \zeta} X_{t} \text { exists in } E, \\ \partial, & \text { otherwise. }\end{cases}
$$

LEMMA 2.13. Let $X$ and $\widehat{X}$ be a pair of standard processes in weak duality with respect to $m$ under the assumption (A.1), and $F$ be a q.e. finely closed subset of $E$ that is not m-polar. For this lemma, we adopt the same notation as in Lemma 2.11, that is, we use $X_{t-}^{0}$ and $X_{t-}$ to denote the left limits under the original topology on $E$ and under the Ray topology, respectively. Define the debut time $D_{F}$ of $F$ by

$$
D_{F}:=\inf \left\{t \geq 0: X_{t} \in F\right\}
$$

and define

$$
R_{F}^{0}:=\inf \left\{t \geq 0: X_{t} \in F \text { or } X_{t-}^{0} \in F\right\} .
$$

Here in the definition of $R_{F}^{0}, X_{\zeta-}^{0}$ is defined in the same way as in (2.19). Then for q.e. $x \in E, D_{F}=R_{F}^{0} \mathbf{P}_{x}$-a.s. Moreover, with $\eta:=\sup \left\{t>0: X_{t} \in\right.$ $F\}$,

$$
\mathbf{P}_{x}\left(\eta<\zeta \text { and } X_{\zeta-}^{0} \in F\right)=0 \quad \mathbf{P}_{x} \text {-a.s. for q.e. } x \in F .
$$

Proof. By Lemma 2.9, we may assume that $X$ is special standard by removing a properly exceptional set from $E$. Since $F$ is q.e. finely closed and $F \backslash F^{r}$ is a semipolar set of $X$ and hence is $m$-polar by condition (A.1), after removing a properly exceptional set, we may and do assume that $F=F^{r}$. Define $u(x)=\mathbf{E}_{x} e^{-\sigma_{F}}$, which is a 1-excessive function of $X$. By (18.5) of [36], $u$ is nearly Borel measurable under the Ray topology of $X$. In particular, we 
conclude that $F=\{x: u(x)=1\}$ is nearly Borel measurable under the Ray topology. According to (12.14) of [20],

$$
D_{F}=R_{F} \quad \mathbf{P}_{x^{-}} \text {-a.s. for every } x \in E,
$$

where $R_{F}$ is defined in the same way as $R_{F}^{0}$ but with $X_{t-}$ in place of $X_{t-}^{0}$. It now follows from $[36,(47.10)]$ and Lemma 2.11 that $D_{F}=R_{F}^{0} \mathbf{P}_{x}$-a.s. for every $x \in E$. Since $F=F^{r}$, we in fact have for every $x \in E, \mathbf{P}_{x^{-}}$a.s.,

$$
\inf \left\{t>0: X_{t} \in F \text { or } X_{t-}^{0} \in F\right\}=\sigma_{F}:=\inf \left\{t>0: X_{t} \in F\right\} \text {. }
$$

It follows, after applying the above to the time shift operator $\theta_{s}$, that for every rational $s>0$ and with $\mathbf{P}_{x}$-a.s. for every $x \in E$,

$$
\inf \left\{t>s: X_{t} \in F \text { or } X_{t-}^{0} \in F\right\}=\inf \left\{t>s: X_{t} \in F\right\} .
$$

This proves $(2.20)$.

Using (2.21), we can identify the random homogeneous set $M(\omega)$ defined by (2.7) with the one that typically appears in the literature for exit systems.

Proposition 2.14. Let $X$ and $\widehat{X}$ be a pair of standard processes in weak duality with respect to $m$ under the assumption (A.1), and $F$ be a q.e. finely closed subset of $E$ that is not m-polar. Then for q.e. $x \in E, \mathbf{P}_{x}$-a.s.

$$
M(\omega)=[0, \zeta(\omega)) \cap \overline{\left\{t \geq 0: X_{t}(\omega) \in F\right\}} .
$$

Proof. By Lemma 2.2, we know that q.e. $x \in E, \mathbf{P}_{x}$-a.s.

$$
M(\omega) \supset[0, \zeta(\omega)) \cap \overline{\left\{t \geq 0: X_{t}(\omega) \in F\right\}} .
$$

So it suffices to show that $M(\omega)$ is contained in the right hand side of (2.23). For this, just as in the proof of Lemma 2.13, we may and do assume that $F=F^{r}$. Note that in this proof, $X_{t-}$ denotes the left limit of $X$ in the original topology of $E$. Let $\Omega_{0}$ be the collection of all $\omega$ so that (2.21) is true. Fix $\omega \in \Omega_{0}$. For $0<t \in M(\omega)$, let $\left\{t_{k}, k \geq 1\right\}$ be a sequence of rational numbers that strictly increases to $t$. It follows from (2.21) that for every $k \geq 1$,

$$
\inf \left\{s>t_{k}: X_{s}(\omega) \in F\right\}=\inf \left\{s>t_{k}: X_{s}(\omega) \in F \text { or } X_{s-}(\omega) \in F\right\} \in\left[t_{k}, t\right] .
$$

Letting $k \rightarrow \infty$ shows that $t \in[0, \zeta(\omega)) \cap \overline{\left\{t \geq 0: X_{t}(\omega) \in F\right\}}$, which proves (2.23).

\section{Entrance law, exit system and Feller measures}

Let $E, m$ be as in $\S 2$ and consider two Borel standard processes $X=$ $\left(X_{t}, \zeta, \mathbf{P}_{x}\right)$ and $\widehat{X}=\left(\widehat{X}_{t}, \widehat{\zeta}, \widehat{\mathbf{P}}_{x}\right)$ on $E$ in weak duality with respect to the measure $m$. We assume for $X$ the condition (A.1) as in the last three subsections. 
We fix a set $F \in \mathcal{B}^{n}$ and put

$$
E_{0}:=E \backslash F, \quad m_{0}:=\left.m\right|_{E_{0}}, \quad(u, v)_{0}:=\int_{E_{0}} u(x) v(x) m_{0}(d x) .
$$

We then assume:

(A.2) $F$ is q.e. finely closed.

(A.3) $\mathbf{P}_{x}\left(\sigma_{F}<\infty\right)>0$, for $m_{0}$-a.e. $x \in E_{0}$.

Let $X^{0}=\left(X_{t}^{0}, \zeta^{0}, \mathbf{P}_{x}\right)_{x \in E^{0}}$ be the subprocess of $X$ killed upon leaving $F$, that is, with $\zeta^{0}=\zeta \wedge \sigma_{F}$,

$$
X_{t}^{0}:= \begin{cases}X_{t} & \text { if } t \in\left[0, \zeta^{0}\right), \\ \partial, & \text { if } t \geq \zeta^{0},\end{cases}
$$

where $\partial$ is an extra point added to $E_{0}$. Denote by $P_{t}^{0}, G_{\alpha}^{0}$ the transition function and the resolvent of $X^{0}$. In particular, $P_{t}^{0} f(x)=\mathbf{E}_{x}\left[f\left(X_{t}\right) ; t<\sigma_{F}\right], x \in$ $E_{0}, f \in \mathcal{B}\left(E_{0}\right)$, and we have from (2.3)

$$
\left(\widehat{P}_{t}^{0} f, g\right)_{0}=\left(f, P_{t}^{0} g\right)_{0}, \quad\left(\widehat{G}_{\alpha}^{0} f, g\right)_{0}=\left(f, G_{\alpha}^{0} g\right)_{0}, \quad f, g \in \mathcal{B}^{+}\left(E_{0}\right) .
$$

The assumptions (A.1) and (A.2) imply that $F$ and $F^{r}$ are q.e. equivalent as was observed in the proof of Lemma 2.2 .

The assumption (A.3) together with (3.1) implies as in [17, Lemma 1.6.5] that $G_{0+}^{0} f(x)<\infty$ for some strictly positive $f \in \mathcal{B}_{b}\left(E_{0}\right) m$-a.e. on $E_{0}$ and hence q.e. on $E_{0}$ by (iii) in $\S 2.1$.

Furthermore, by Lemma 2.2, the random set $M(\omega)$ defined for $F$ by $(2.7)$ is a relatively closed subset of $[0, \zeta(\omega))$, and moreover, if $t_{n} \in M(\omega)$ increase to $t<\zeta$, then $X_{t-}(\omega) \in F, \mathbf{P}_{x}$-a.s. for q.e. $x \in E$.

Therefore, by (iv) in $\S 2.1$, there exists a properly exceptional set $N \subset E$ such that, denoting $E \backslash N, F \backslash N, E_{0} \backslash N$ and the restriction $\left.X\right|_{E \backslash N}$ by $E, F, E$ and $X$, respectively, again, we can assume from the beginning that the following properties hold.

(I) $F=F^{r}$.

(II) $X^{0}$ is transient: there is a strictly positive $f \in \mathcal{B}_{b}\left(E_{0}\right)$ on $E_{0}$ such that $G_{0+}^{0} f(x)<\infty$ for every $x \in E_{0}$.

(III) $M(\omega)$ is relatively closed in $[0, \zeta(\omega))$, and furthermore, if $t_{n} \in M(\omega)$ increase to $t<\zeta$, then $X_{t-}(\omega) \in F, \mathbf{P}_{x^{-}}$-a.s. for every $x \in E$.

As in $\S 2$, we consider, for $f \in \mathcal{B}^{+}(E), \alpha>0$ and $x \in E$,

$$
\mathbf{H} f(x)=\mathbf{E}_{x}\left[f\left(X_{\sigma_{F}}\right) ; \sigma_{F}<\infty\right] \quad \text { and } \quad \mathbf{H}_{\alpha} f(x)=\mathbf{E}_{x}\left[e^{-\alpha \sigma_{F}}\left(X_{\sigma_{F}}\right)\right] \text {, }
$$

together with the corresponding notations $\widehat{\mathbf{H}}, \widehat{\mathbf{H}}_{\alpha}$ for $\widehat{X}$.

Since

$$
\widehat{P}_{t}^{0} \widehat{\mathbf{H}} f(x)=\widehat{\mathbf{E}}_{x}\left[f\left(\widehat{X}_{\sigma_{F}}\right) ; t<\sigma_{F}\right] \leq \widehat{\mathbf{H}} f(x), \quad x \in E_{0},
$$

we see from (3.1) that the measure $\widehat{\mathbf{H}} f \cdot m_{0}$ is $X^{0}$-excessive for any $f \in \mathcal{B}_{b}^{+}(E)$. 
In accordance with [22, Prop. 3.6] (see also [16, Lemma 2.1]), the energy functional $L^{(0)}(\eta, u)$ of an $X^{0}$-excessive measure $\eta$ on $E_{0}$ and an $X^{0}$-excessive function $u$ on $E_{0}$ (with respect to $X^{0}$ ) is well defined by

$$
L^{(0)}(\eta, u):=\uparrow \lim _{t \downarrow 0} \frac{1}{t}\left\langle\eta-\eta P_{t}^{0}, u\right\rangle .
$$

We can then define a bimeasure $U$ on $E \times E$ by

$$
U(f \otimes g):=L^{(0)}\left(\widehat{\mathbf{H}} f \cdot m_{0}, \mathbf{H} g\right)=\uparrow \lim _{t \downarrow 0} \frac{1}{t}\left(\widehat{\mathbf{H}} f, \mathbf{H} g-P_{t}^{0} \mathbf{H} g\right), \quad f, g \in \mathcal{B}_{b}^{+}(E) .
$$

We call $U$ the Feller measure. Actually $U$ charges on $\widehat{F} \times F$, where $\widehat{F}$ denotes the co-fine closure of $F$. We also define the supplementary Feller measure $V$ by

$$
V(f)=L\left(\widehat{\mathbf{H}} f \cdot m_{0}, q\right) \quad \text { for } f \in \mathcal{B}_{b}^{+}(E),
$$

where $q(x):=1-\mathbf{H} 1(x)=\mathbf{P}_{x}\left(\sigma_{F}=\infty\right)$. The measure $V$ charges on $\widehat{F}$.

We call a family of $\sigma$-finite measures $\left\{\nu_{t}, T>0\right\}$ on $E_{0}$ an $X^{0}$-entrance law if

$$
\nu_{t} p_{s}^{0}=\nu_{t+s} \quad \text { for } t, s>0 .
$$

The next lemma is an extension of Lemma 2.2 of [18].

LEMMA 3.1.

(i) For any $f \in \mathcal{B}_{b}^{+}(E)$, there exists a unique $X^{0}$-entrance law $\mu_{t}^{f}$ such that

$$
\widehat{\mathbf{H}} f \cdot m_{0}=\int_{0}^{\infty} \mu_{t}^{f} d t .
$$

(ii) Denote by $\mu_{\alpha}^{\# f}$ the Laplace transform of $\mu_{t}^{f}$. Then, for any $v \in \mathcal{B}^{+}\left(E_{0}\right)$,

$$
\int_{t}^{\infty}\left\langle\mu_{s}^{f}, v\right\rangle d s=\left(\widehat{\mathbf{H}} f, P_{t}^{0} v\right)_{0} \quad \text { for every } t>0
$$

and

$$
\left\langle\mu_{\alpha}^{\# f}, v\right\rangle=\left(\widehat{\mathbf{H}}_{\alpha} f, v\right)_{0} .
$$

(iii) For any $f \in \mathcal{B}_{b}^{+}(E)$ and $X^{0}$-excessive function $v$ on $E_{0},\left\langle\mu_{t}^{f}, v\right\rangle$ is right continuous, decreasing in $t>0$ and

$$
L^{(0)}\left(\widehat{\mathbf{H}} f \cdot m_{0}, v\right)=\uparrow \lim _{t \downarrow 0}\left\langle\mu_{t}^{f}, v\right\rangle .
$$

In particular,

$$
U(f, g)=\uparrow \lim _{t \downarrow 0}\left\langle\mu_{t}^{f}, \mathbf{H} g\right\rangle \quad \text { and } \quad V(f)=\uparrow \lim _{t \downarrow 0}\left\langle\mu_{t}^{f}, q\right\rangle .
$$


Proof. The proof of this lemma is analogous to the proof of Lemma 2.2 of [18], but we spell out its proof for completeness.

(i) By (3.1) and (3.2),

$$
\lim _{t \rightarrow \infty}\left\langle\left(\widehat{\mathbf{H}} f \cdot m_{0}\right) P_{t}^{0}, v\right\rangle=\lim _{t \rightarrow \infty}\left(\widehat{P}_{t}^{0} \widehat{\mathbf{H}} f, v\right)_{0}=0 \quad \text { for } v \in L^{1}\left(E_{0}, m_{0}\right),
$$

that is, $\widehat{\mathbf{H}} f \cdot m_{0}$ is purely excessive. Since $X^{0}$ is transient (see (II)), the assertion follows from [22, Th. 5.21] (see also [7]).

(ii) For $v \in L^{1}\left(E_{0} ; m_{0}\right)$, we have

$$
\begin{aligned}
\int_{t}^{\infty}\left\langle\mu_{t}^{f}, v\right\rangle d t & =\int_{0}^{\infty}\left\langle\mu_{t+s}^{f}, v\right\rangle d t=\int_{0}^{\infty}\left\langle\mu_{s}^{f}, P_{t}^{0} v\right\rangle d s \\
& =\left(\widehat{\mathbf{H}} f \cdot m_{0}, P_{t}^{0} v\right\rangle=\left(\widehat{\mathbf{H}} f, P_{t}^{0} v\right)_{0},
\end{aligned}
$$

and

$$
\left\langle\mu_{t}^{f}, v\right\rangle=-\frac{d}{d t}\left(\widehat{\mathbf{H}} f, P_{t}^{0} v\right)_{0}, \quad \text { a.e. } t .
$$

Hence

$$
\begin{aligned}
\left\langle\mu_{\alpha}^{\# f}, v\right\rangle & =-\int_{0}^{\infty} e^{-\alpha t} \frac{d}{d t}\left(\widehat{\mathbf{H}} f, P_{t}^{0} v\right)_{0} d t \\
& =\left[-e^{-\alpha t}\left(\widehat{\mathbf{H}} f, P_{t}^{0} v\right)_{0}\right]_{0}^{\infty}-\alpha \int_{0}^{\infty} e^{-\alpha t}\left(\widehat{\mathbf{H}} f, P_{t}^{0} v\right)_{0} d t \\
& =(\widehat{\mathbf{H}} f, v)_{0}-\alpha\left(\widehat{\mathbf{H}} f, G_{\alpha}^{0} v\right)_{0} \\
& =\left(\widehat{\mathbf{H}} f-\alpha \widehat{G}_{\alpha}^{0} \widehat{\mathbf{H}} f, v\right)_{0}=\left(\widehat{\mathbf{H}}_{\alpha} f, v\right)_{0} .
\end{aligned}
$$

(iii) If $v$ is $X^{0}$-excessive, then $\left\langle\mu_{t+s}, v\right\rangle=\left\langle\mu_{t}, P_{t}^{0} v\right\rangle \uparrow\left\langle\mu_{t}, v\right\rangle$ as $s \downarrow 0$. For $v \in L^{1}\left(E_{0} ; m_{0}\right)$, we get from (i) and (ii) that

$$
\left\langle\widehat{\mathbf{H}} f \cdot m_{0}-\left(\widehat{\mathbf{H}} f \cdot m_{0}\right) P_{t}^{0}, v\right\rangle=\int_{0}^{t}\left\langle\mu_{s}^{f}, v\right\rangle d s,
$$

which extends to any $X^{0}$-excessive $v$ and leads us to the desired identity.

Recall the random set $M(\omega)$ defined by $(2.7)$ for $F$. It is a relatively closed subset of $[0, \zeta(\omega))$ almost surely by (III) of $\S 3$. Let $I$ denote the left endpoints for each component of the relatively open set $[0, \zeta(\omega)) \backslash M(\omega)$. This set consists of the "excursion intervals" away from $F$ of the sample path $X(\omega)$. Clearly $M$ is homogeneous on $(0, \infty)$, i.e., for every $s \geq 0$,

$$
(M-s) \cap(0, \infty)=\left(M \circ \theta_{s}\right) \cap(0, \infty)
$$

and so is $I$.

Define

$$
R(\omega)=\inf \{s>0: s \in M(\omega)\}
$$


Since $X$ and $\widehat{X}$ is a pair of standard processes in weak duality, we have by (2.21) above or by [24, Proposition 15.7] that for q.e. $x \in E, \mathbf{P}_{x}$-almost surely

$$
\begin{aligned}
\sigma_{F} & :=\inf \left\{t>0: X_{t} \in F\right\} \\
& =\inf \left\{t \in(0, \zeta): X_{t} \in F \text { or } X_{t-} \in F\right\},
\end{aligned}
$$

and so

$$
R=\sigma_{F} \quad \mathbf{P}_{x^{-a} \text {-a.s. for q.e. } x \in E .}
$$

By enlarging the properly exceptional set $N \subset E$ appearing in the paragraph preceding (I)-(III), we may assume that (3.9) holds $\mathbf{P}_{x}$-a.s. for every $x \in E$. This, combined with the property (I), implies the identity

$$
F=\left\{x \in E: \mathbf{P}_{x}(R=0)=1\right\} .
$$

Let $\left(\mathbf{P}_{x}^{*}, K+J\right)$ be an exit system in the sense of Maisonneuve [31, (4.11)]. That is, $K$ is a PCAF of $X$ carried on $F, d J_{t}=\sum_{s \in I: X_{s} \in E \backslash F} \varepsilon_{s}(d t)$, and $\mathbf{P}^{*}$ is a kernel from $E$ to $\Omega$ such that

$$
\mathbf{E}_{x}\left[\sum_{s \in I} Z_{s} \cdot \Gamma \circ \theta_{s}\right]=\mathbf{E}_{x}\left[\int_{0}^{\infty} Z_{s} \cdot \mathbf{P}_{X_{s}}^{*}(\Gamma) d\left(K_{s}+J_{s}\right)\right], \quad x \in E,
$$

for any positive optional process $Z_{s}$ and positive r.v. $\Gamma$. Here $\varepsilon_{s}(d t)$ is the unit atomic measure on $\mathbb{R}$ concentrated at the point $\{s\}$. Note that, for $x \in E_{0}$, $\mathbf{P}_{x}^{*}$ is defined to be $\mathbf{P}_{x} . \mathbf{P}_{x}^{*}$ also satisfies

$$
\mathbf{P}_{x}^{*}(\zeta \wedge R=0)=0 \quad \text { and } \quad \mathbf{E}_{x}^{*}\left[1-e^{-R}\right] \leq 1 \quad \text { for } x \in E .
$$

REMARK 3.2. In Maisonneuve [31], the exit system is constructed for a conservative strong Markov process $X$ that satisfies the condition that its excessive functions are nearly Borel and right continuous along the sample paths of $X$. In our setting, the Borel standard process $X$ may have finite lifetime. However, the results of Maisonneuve [31] are applicable here since we can regard the cemetery point $\partial$ as the absorbing state of $X$.

Denote by $Q_{t}^{*}(x, \cdot), x \in F$, the entrance law with respect to $\mathbf{P}_{x}^{*}$ defined by $[31,(6.2)]$ :

$$
Q_{t}^{*} g(x):=\mathbf{E}_{x}^{*}\left[g\left(X_{t}\right) ; t<R\right] \quad \text { for } t>0, x \in E, g \in \mathcal{B}^{+}(E) .
$$

Note that $Q_{t}^{*} g(x)=P_{t}^{0} g(x)$ for $x \in E_{0}$.

On account of Lemma 2.9 and Lemma 2.12, $X$ is an $m$-special standard process and has a Lévy system $(N, H)$ in the following sense: $N(x, d y)$ is a kernel on $\left(E_{\partial}, \mathcal{B}\left(E_{\partial}\right)\right)$ and $H$ is a PCAF $H$ of $X$ with bounded 1-potential such that for any nonnegative Borel function $f$ on $E \times E_{\partial}$ that vanishes on the diagonal and is extended to be zero elsewhere,

$$
\mathbf{E}_{x}\left[\sum_{s \leq t} f\left(X_{s-}, X_{s}\right)\right]=\mathbf{E}_{x}\left[\int_{0}^{t} \int_{E_{\partial}} f\left(X_{s}, y\right) N\left(X_{s}, d y\right) d H_{s}\right]
$$


for q.e. $x \in E$ and $t \geq 0$, where $X_{\zeta-}$ is defined by (2.19). The Revuz measure of $H$ with respect to the excessive measure $m$ will be denoted by $\mu_{H}$.

THEOREM 3.3. For any Borel subset $B \subset E_{0}$ and $f \in \mathcal{B}_{b}(F)$,

$$
\mu_{t}^{f}(B)=\int_{F} f(x) Q_{t}^{*}(x, B) \mu_{K}(d x)+\int_{F \times(E \backslash F)} f(x) P_{t}^{0} 1_{B}(y) N(x, d y) \mu_{H}(d x),
$$

where $\mu_{K}$ is the Revuz measure of the PCAF K with respect to the measure $m$.

Proof. Put

$$
\mathbf{Q}_{\alpha}^{*} g(x)=\int_{0}^{\infty} e^{-\alpha t} Q_{t}^{*} g(x) d t, \quad g \in \mathcal{B}_{b}(E) .
$$

By virtue of the identity (3.8), we have for any $v \in \mathcal{B}_{b}(E)$ vanishing on $F$ and for every $x \in E$,

$$
\begin{aligned}
\mathbf{H}_{\alpha} G_{\alpha} v(x) & =G_{\alpha} v(x)-G_{\alpha}^{0} v(x) \\
& =\mathbf{E}_{x}\left[\int_{R}^{\infty} e^{-\alpha t} v\left(X_{t}\right) 1_{M^{c}}(t) d t\right] \\
& =\mathbf{E}_{x}\left[\sum_{s \in I} \int_{s}^{s+R \circ \theta_{s}} e^{-\alpha t} v\left(X_{t}\right) d t\right] \\
& =\mathbf{E}_{x}\left[\sum_{s \in I} e^{-\alpha s} \int_{0}^{R} e^{-\alpha t} v\left(X_{t}\right) d t \circ \theta_{s}\right] \\
& =\mathbf{E}_{x}\left[\int_{0}^{\infty} e^{-\alpha s} \mathbf{E}_{X_{s}}^{*}\left[\int_{0}^{R} e^{-\alpha t} v\left(X_{t}\right) d t\right] d\left(K_{s}+J_{s}\right)\right] .
\end{aligned}
$$

Therefore we have for any $f \in \mathcal{B}_{b}^{+}(E)$,

$$
\left(f, \mathbf{H}_{\alpha} G_{\alpha} v\right)=\mathbf{E}_{f \cdot m}\left[\int_{0}^{\infty} e^{-\alpha s} \mathbf{Q}_{\alpha}^{*} v\left(X_{s}\right) d\left(K_{s}+J_{s}\right)\right] .
$$

On the other hand, owing to the fundamental Revuz formula (2.13), we obtain

$$
\mathbf{E}_{f \cdot m}\left[\int_{0}^{\infty} e^{-\alpha s} \mathbf{Q}_{\alpha}^{*} v\left(X_{s}\right) d K_{s}\right]=\left\langle\mathbf{Q}_{\alpha}^{*} v \cdot \mu_{K}, \widehat{G}_{\alpha} f\right\rangle .
$$

Furthermore, since $\mathbf{Q}_{\alpha}^{*}(x, \cdot)=G_{\alpha}^{0}(x, \cdot)$ for $x \in E_{0}$, we have 


$$
\begin{aligned}
\mathbf{E}_{f \cdot m} & {\left[\int_{0}^{\infty} e^{-\alpha s} \mathbf{Q}_{\alpha}^{*} v\left(X_{s}\right) d J_{s}\right] } \\
& =\mathbf{E}_{f \cdot m}\left[\sum_{s \in I, X_{s} \in E \backslash F} e^{-\alpha s} G_{\alpha}^{0} v\left(X_{s}\right)\right] \\
& =\mathbf{E}_{f \cdot m}\left[\sum_{s} e^{-\alpha s} 1_{F}\left(X_{s-}\right) 1_{E \backslash F}\left(X_{s}\right) G_{\alpha}^{0} v\left(X_{s}\right)\right] \\
& =\mathbf{E}_{f \cdot m}\left[\int_{0}^{\infty} e^{-\alpha s} 1_{F}\left(X_{s}\right) \int_{E \backslash F} N\left(X_{s}, d z\right) G_{\alpha}^{0} v(z) d H_{s}\right] \\
& =\left\langle 1_{F} \cdot \int_{E \backslash F} N(\cdot, d z) G_{\alpha}^{0} v(z) \cdot \mu_{H}, \widehat{G}_{\alpha} f\right\rangle .
\end{aligned}
$$

Here in the last equality we used (2.13) again.

Applying the duality relation (2.4), we get from (3.13), (3.14) and (3.15)

$$
\left(\widehat{\mathbf{H}}_{\alpha} \widehat{G}_{\alpha} f, v\right)=\left\langle\mathbf{Q}_{\alpha}^{*} v \cdot \mu_{K}+1_{F}(\cdot) \int_{E \backslash F} N(\cdot, d z) G_{\alpha}^{0} v(z) \cdot \mu_{H}, \widehat{G}_{\alpha} f\right\rangle .
$$

Since this identity holds for an arbitrary $f \in \mathcal{B}_{b}(E)$, we obtain for any $\beta>0$, $f \in C_{b}^{+}(E) \cap \mathcal{F}$ and $v \in L^{1}\left(E_{0}, m\right)$,

$$
\left(\widehat{\mathbf{H}}_{\alpha} \widehat{G}_{\beta} f, v\right)=\left\langle\mathbf{Q}_{\alpha}^{*} v \cdot \mu_{K}+1_{F}(\cdot) \int_{E \backslash F} N(\cdot, d z) G_{\alpha}^{0} v(z) \cdot \mu_{H}, \widehat{G}_{\beta} f\right\rangle .
$$

Multiplying $\beta$ on both sides of (3.16) with $f=1$ and then letting $\beta \rightarrow \infty$, we have by the monotone convergence theorem,

$$
\left\langle\mathbf{Q}_{\alpha}^{*} v \cdot \mu_{K}+1_{F} \cdot \int_{E \backslash F} N(\cdot, d z) G_{\alpha}^{0} v(z) \cdot \mu_{H}, 1\right\rangle=\left\langle\widehat{\mathbf{H}}_{\alpha} 1, v\right\rangle \leq \int_{E_{0}} v(x) m(d x)<\infty .
$$

Now multiplying both sides of (3.16) by $\beta$ and letting $\beta \rightarrow \infty$ in the above equation, we have by the bounded convergence theorem,

$$
\left(\widehat{\mathbf{H}}_{\alpha} f, v\right)=\left\langle\mathbf{Q}_{\alpha}^{*} v \cdot \mu_{K}+1_{F}(\cdot) \int_{E \backslash F} N(\cdot, d z) G_{\alpha}^{0} v(z) \cdot \mu_{H}, f\right\rangle .
$$

This combined with (3.7) proves the desired identity (3.12) since the above display is nothing but the Laplace transform of (3.12).

Theorem 3.3 allows us to make the connection between Feller measures $U$ and $V$ with the exit system $\left(\mathbf{P}_{x}^{*}, K+J\right)$.

Theorem 3.4. The Feller measure $U$ is carried by $F \times F$ and

$$
\begin{aligned}
U(d x, d y)=\mu_{K}(d x) \mathbf{P}_{x}^{*}\left(X_{\sigma_{F}} \in d y\right) & \\
& +\left.\mu_{H}(d x)\right|_{F} \int_{E \backslash F} N(x, d z) \mathbf{P}_{z}\left(X_{\sigma_{F}} \in d y\right) .
\end{aligned}
$$


The supplementary Feller measure $V$ is carried by $F$ and

$$
\begin{aligned}
& V(d x)=\mu_{K}(d x) \mathbf{P}_{x}^{*}\left(\zeta>0, \sigma_{F}=\infty\right) \\
& \quad+\left.\mu_{H}(d x)\right|_{F} \int_{E \backslash F} N(x, d z) \mathbf{P}_{z}\left(\sigma_{F}=\infty\right)
\end{aligned}
$$

Proof. It follows from $(3.8),(3.12)$ and the definition of $Q_{t}^{*}(x, d y)$ that for any $f, g \in \mathcal{B}^{+}(E)$,

$$
\begin{aligned}
& \int_{F \times F} f(x) g(y) U(d x, d y)=\lim _{t \downarrow 0}\left\langle\mu_{t}^{f}, \mathbf{H} g\right\rangle \\
& =\lim _{t \downarrow 0}\left[\int_{F \times E_{0}} f(x) \mathbf{H} g(y) Q_{t}^{*}(x, d y) \mu_{K}(d x)\right. \\
& \left.\quad+\int_{F \times E_{0}} f(x) P_{t}^{0} \mathbf{H} g(y) N(x, d y) \mu_{H}(d x)\right] \\
& \quad \lim _{t \downarrow 0} \int_{F} f(x) \mathbf{E}_{x}^{*}\left[\mathbf{H} g\left(X_{t}\right) ; t<R\right] \mu_{K}(d x)+\int_{F \times E_{0}} f(x) \mathbf{H} g(y) N(x, d y) \mu_{H}(d x) \\
& =\lim _{t \downarrow 0} \int_{F} f(x) \mathbf{E}_{x}^{*}\left[g\left(X_{\sigma_{F}}\right) \circ \theta_{t} ; t<R\right] \mu_{K}(d x) \\
& \quad+\int_{F \times E_{0}} f(x) \mathbf{H} g(y) N(x, d y) \mu_{H}(d x) \\
& =\lim _{t \downarrow 0} \int_{F} f(x) \mathbf{E}_{x}^{*}\left[g\left(X_{\sigma_{F}}\right) ; t<R\right] \mu_{K}(d x)+\int_{F \times E_{0}} f(x) \mathbf{H} g(y) N(x, d y) \mu_{H}(d x) \\
& =\int_{F} f(x) \mathbf{E}_{x}^{*}\left[g\left(X_{\sigma_{F}}\right)\right] \mu_{K}(d x)+\int_{F \times E_{0}} f(x) \mathbf{E}_{y}\left[g\left(X_{\sigma_{F}}\right)\right] N(x, d y) \mu_{H}(d x) .
\end{aligned}
$$

In the third through the last equalities, we used the strong Markov property for the excursion measure $\mathbf{P}^{*}$ (see Theorem 5.1 of [31]). Identity (3.17) now follows.

The proof for (3.18) is similar to that for (3.17). For completeness, we spell out the details. Note that for $x \in E_{0}, q(x)=1-\mathbf{H} 1(x)=\mathbf{P}_{x}\left(\sigma_{F}=\infty\right)$. Thus by (3.8), for any $f \in \mathcal{B}^{+}(E)$,

$$
\begin{aligned}
& \int_{F} f(x) V(d x)=\lim _{t \downarrow 0}\left\langle\mu_{t}^{f}, q\right\rangle \\
& =\lim _{t \downarrow 0}\left[\int_{F \times E_{0}} f(x) q(y) Q_{t}^{*}(x, d y) \mu_{K}(d x)+\int_{F \times E_{0}} f(x) P_{t}^{0} q(y) N(x, d y) \mu_{H}(d x)\right]
\end{aligned}
$$




$$
\begin{aligned}
& =\lim _{t \downarrow 0} \int_{F} f(x) \mathbf{E}_{x}^{*}\left[q\left(X_{t}\right) ; t<R\right] \mu_{K}(d x)+\int_{F \times E_{0}} f(x) q(y) N(x, d y) \mu_{H}(d x) \\
& =\lim _{t \downarrow 0} \int_{F} f(x) \mathbf{E}_{x}^{*}\left[1_{\left\{\sigma_{F}=\infty\right\}} \circ \theta_{t} ; t<\zeta \wedge R\right] \mu_{K}(d x) \\
& \quad \quad+\int_{F \times E_{0}} f(x) q(y) N(x, d y) \mu_{H}(d x) \\
& =\int_{F} f(x) \mathbf{P}_{x}^{*}\left(\zeta>0, \sigma_{F}=\infty\right) \mu_{K}(d x) \\
& \quad+\int_{F \times E_{0}} f(x) \mathbf{P}_{y}\left(\sigma_{F}=\infty\right) N(x, d y) \mu_{H}(d x) .
\end{aligned}
$$

This establishes (3.18). Since $\mu_{K}$ charges on $F$, we conclude from the expressions (3.17) and (3.18) that the measures $U$ and $V$ charge on $F \times F$ and $F$, respectively.

The next corollary relates the Feller measures to the distributions of end places of excursions:

Corollary 3.5. For every $\Psi \in \mathcal{B}^{+}(E \times E \backslash d)$ and $f \in \mathcal{B}^{+}(E)$ that are extended to be zero off $E \times E \backslash d$ and $E$, respectively, we have

$$
\int_{F \times F} \Psi(x, y) U(d x, d y)=\lim _{t \downarrow 0} \frac{1}{t} \mathbf{E}_{m}\left[\sum_{s \in I, s \leq t} \Psi\left(X_{s-}, X_{\sigma_{F}} \circ \theta_{s}\right)\right]
$$

and

$$
\int_{F} f(\xi) V(d \xi)=\lim _{t \downarrow 0} \frac{1}{t} \mathbf{E}_{m}\left[\sum_{s \in I, s \leq t} f\left(X_{s-}\right) 1_{\left\{\zeta>0, \sigma_{F}=\infty\right\}} \circ \theta_{s}\right]
$$

Proof. Take any positive measurable functions $f, g$ on $E$ and put

$$
Z_{s}=f\left(X_{s-}\right) 1_{(0, t]}(s) \quad \Lambda=g\left(X_{\sigma_{F}}\right) .
$$

By the formula (3.10), we then have 


$$
\begin{aligned}
& \lim _{t \downarrow 0} \frac{1}{t} \mathbf{E}_{m}\left[\sum_{s \in I \cap[0, \zeta), s \leq t} f\left(X_{s-}\right) g\left(X_{\sigma_{F}} \circ \theta_{s}\right)\right] \\
& =\lim _{t \downarrow 0} \frac{1}{t} \mathbf{E}_{m}\left[\int_{0}^{t} f\left(X_{s-}\right) \mathbf{E}_{X_{s}}^{*}\left[g\left(X_{\sigma_{F}}\right)\right] d K_{s}\right] \\
& \quad+\lim _{t \downarrow 0} \frac{1}{t} \mathbf{E}_{m}\left[\sum_{s \in I, X_{s} \in E \backslash F, s \leq t} f\left(X_{s-}\right) \mathbf{E}_{X_{s}}\left[g\left(X_{\sigma_{F}}\right)\right]\right] \\
& =\int_{E} f(x) \mathbf{E}_{x}^{*}\left[g\left(X_{\sigma_{F}}\right)\right] \mu_{K}(d x)+\lim _{t \downarrow 0} \frac{1}{t} \mathbf{E}_{m}\left[\sum_{s \leq t}\left(1_{F} f\right)\left(X_{s-}\right) 1_{E \backslash F}\left(X_{s}\right) \mathbf{H} g\left(X_{s}\right)\right] \\
& =\int_{E} f(x) \mathbf{E}_{x}^{*}\left[g\left(X_{\sigma_{F}}\right)\right] \mu_{K}(d x) \\
& \quad+\lim _{t \downarrow 0} \frac{1}{t} \mathbf{E}_{m}\left[\int_{0}^{t}\left(1_{F} f\right)\left(X_{s}\right) \int_{E_{0}} \mathbf{H} g(z) N\left(X_{s}, d z\right) d H_{s}\right] \\
& =\int_{F \times F} f(x) g(y) \mathbf{P}_{x}^{*}\left(X_{\sigma_{F}} \in d y\right) \mu_{K}(d x)+\int_{F \times E_{0}} f(x) \mathbf{H} g(z) N(x, d z) \mu_{H}(d x),
\end{aligned}
$$

which, together with (3.17), leads us to (3.19) for $\Psi=f \otimes g$. A monotone class argument establishes (3.19) for general $\Psi \geq 0$ on $F \times F$.

Again by (3.10),

$$
\begin{aligned}
& \lim _{t \downarrow 0} \frac{1}{t} \mathbf{E}_{m}\left[\sum_{s \in I} f\left(X_{s-t}\right) 1_{\left\{\sigma_{F}=\infty\right\}} \circ \theta_{s}\right] \\
& =\lim _{t \downarrow 0} \frac{1}{t} \mathbf{E}_{m}\left[\sum_{s \in I s \leq t} f\left(X_{s-}\right) 1_{\left\{\zeta>0 . \sigma_{F}=\infty\right\}} \circ \theta_{s}\right] \\
& =\lim _{t \downarrow 0} \frac{1}{t} \mathbf{E}_{m}\left[\int_{0}^{t} f\left(X_{s-}\right) \mathbf{P}_{X_{s}}^{*}\left(\zeta>0, \sigma_{F}=\infty\right) d K_{s}\right] \\
& \quad+\lim _{t \downarrow 0} \frac{1}{t} \mathbf{E}_{m}\left[\sum_{s \in I, X_{s} \in E \backslash F, s \leq t} f\left(X_{s-}\right) \mathbf{P}_{X_{s}}\left(\sigma_{F}=\infty\right)\right] \\
& =\int_{F} f(x) \mathbf{P}_{x}^{*}\left(\zeta>0, \sigma_{F}=\infty\right) \mu_{K}(d x) \\
& \quad+\lim _{t \downarrow 0} \frac{1}{t} \mathbf{E}_{m}\left[\int_{0}^{t}\left(1_{F} f\right)\left(X_{s}\right) \int_{E_{0}} \mathbf{P}_{y}\left(\sigma_{F}=\infty\right) N\left(X_{s}, d y\right) d H_{s}\right] \\
& =\int_{F} f(x) \mathbf{P}_{x}^{*}\left(\zeta>0, \sigma_{F}=\infty\right) \mu_{K}(d x) \\
& \quad+\int_{F \times E_{0}} f(x) \mathbf{P}_{y}\left(\sigma_{F}=\infty\right) N(x, d y) \mu_{H}(d y),
\end{aligned}
$$


which, together with (3.18), leads us to (3.20).

Corollary 3.5 will be used in $\S 5$.

\section{Feller-Neveu measure}

This section is a continuation of the preceding one. For $\alpha>0$, we define the $\alpha$-order Feller measure $U_{\alpha}$ by

$$
U_{\alpha}(f, g)=\alpha\left(\widehat{\mathbf{H}}_{\alpha} f, \mathbf{H} g\right), \quad f, g \in \mathcal{B}_{b}^{+}(E) .
$$

We then have as in $[16, \S 2]$

$$
\lim _{\alpha \rightarrow \infty} U_{\alpha}(f, g)=U(f, g) .
$$

The notions $U_{\alpha}$ and $U$ go back to [6]. The next theorem is a consequence of Theorem 3.3.

THEOREM 4.1. For each fixed $f, g \in \mathcal{B}_{b}^{+}(F)$, define

$$
\Theta^{f, g}((s, t]):=\left\langle\mu_{s}^{f}, \mathbf{H} g-P_{t-s}^{0} \mathbf{H} g\right\rangle, \quad 0<s \leq t .
$$

This uniquely extends to a measure $\Theta^{f, g}$ on $(0, \infty)$ defined by

$$
\begin{aligned}
\Theta^{f, g}(d u)= & \int_{F} f(x) \mathbf{E}_{x}^{*}\left[g\left(X_{\sigma_{F}}\right) ; \sigma_{F} \in d u\right] \mu_{K}(d x) \\
& +\int_{F \times(E \backslash F)} f(x) \mathbf{E}_{y}\left[g\left(X_{\sigma_{F}}\right) ; \sigma_{F} \in d u\right] N(x, d y) \mu_{H}(d x) .
\end{aligned}
$$

There exist Borel sets $B_{n}$ increasing to $E$ such that $\Theta^{f, 1_{B_{n}}}(d u)$ is $\sigma$-finite on $[0, \infty)$ for each $n$. Furthermore,

$$
U_{\alpha}(f, g)=\int_{0}^{\infty}\left(1-e^{-\alpha u}\right) \Theta^{f, g}(d u), \quad \alpha>0 .
$$

REMARK 4.2. (i) The measure $\Theta^{f, g}$ governs the distribution of the excursion length.

(ii) When $m(E)<\infty$, we see that $\left\langle\mu_{t}^{f}, \mathbf{H} g\right\rangle$ is finite by (3.7), right continuous, decreasing in $t$ by Lemma 3.1 (iii), and

$$
\Theta^{f, g}((s, t])=\left\langle\mu_{s}^{f}, \mathbf{H} g\right\rangle-\left\langle\mu_{t}^{f}, \mathbf{H} g\right\rangle, \quad 0<s<t,
$$

which therefore extends to a unique $\sigma$-finite measure on $(0, \infty)$. In the following proof for the general case, we shall proceed differently. Such a measure that is related to $U_{\alpha}(f, g)$ via (4.5) first appeared in [34], and we therefore we call it the Feller-Neveu measure.

(iii) Letting $\alpha \rightarrow \infty$ in (4.5), we obtain

$$
U(f, g)=\Theta^{f, g}([0, \infty)),
$$

which combined with (4.4) gives another proof of the first half of Theorem 3.4 . 
Proof. Clearly the measure $\Theta^{f, g}(d u)$ given by (4.4) is well-defined. By making use of Theorem 3.3, we first show that $\Theta^{f, g}((s, t])$ defined by $(4.3)$ is the charge of the measure $\Theta^{f, g}$ of (4.4) on the interval $(s, t]$;

$$
\begin{aligned}
\Theta^{f, g}((s, t])= & \int_{F} f(x) \mathbf{E}_{x}^{*}\left[g\left(X_{\sigma_{F}}\right) ; s<\sigma_{F} \leq t\right] \mu_{K}(d x) \\
& +\int_{F \times E_{0}} f(x) \mathbf{E}_{y}\left[g\left(X_{\sigma_{F}}\right) ; s<\sigma_{F} \leq t\right] N(x, d y) \mu_{H}(d x),
\end{aligned}
$$

for $0<s<t$.

Clearly from the definition (4.3),

$$
\Theta^{f, g}((s, t])=\left\langle\mu_{s}^{f}, \mathbf{E} \cdot\left[g\left(X_{\sigma_{F}}\right) ; \sigma_{F} \leq t-s\right]\right\rangle,
$$

which combined with (3.12) leads us to

$$
\begin{aligned}
& \Theta^{f, g}((s, t])=\left\langle\mu_{s}^{f}, \mathbf{E} \cdot\left[g\left(X_{\sigma_{F}}\right) ; \sigma_{F} \leq t-s\right]\right\rangle \\
& =\int_{F} f(x) \mathbf{E}_{y}\left[g\left(X_{\sigma_{F}}\right) ; \sigma_{F} \leq t-s\right] Q_{s}^{*}(x, d y) \mu_{K}(d x) \\
& \quad+\int_{F \times E_{0}} f(x) \mathbf{E}_{y}^{0}\left[\mathbf{E}_{X_{s}}\left[g\left(X_{\sigma_{F}}\right) ; \sigma_{F} \leq t-s\right]\right] N(x, d y) \mu_{H}(d x) \\
& =\int_{F} f(x) \mathbf{E}_{x}^{*}\left[\mathbf{E}_{X_{s}}\left[g\left(X_{\sigma_{F}}\right) ; \sigma_{F} \leq t-s\right] ; s<R\right] \mu_{K}(d x) \\
& \quad+\int_{F \times E_{0}} f(x) \mathbf{E}_{y}\left[g\left(X_{\sigma_{F}}\right) ; s<\sigma_{F} \leq t\right] N(x, d y) \mu_{H}(d x) \\
& =\int_{F} f(x) \mathbf{E}_{x}^{*}\left[g\left(X_{\sigma_{F}}\right) ; s<\sigma_{F} \leq t\right] \mu_{K}(d x) \\
& \quad+\int_{F \times E_{0}} f(x) \mathbf{E}_{y}\left[g\left(X_{\sigma_{F}}\right) ; s<\sigma_{F} \leq t\right] N(x, d y) \mu_{H}(d x) .
\end{aligned}
$$

A comparison of the right hand sides of (3.12) and (4.6) gives

$$
\Theta^{f, g}((s, t]) \leq\left\langle\mu_{s}^{f}, g\right\rangle, \quad 0<s \leq t .
$$

Since $\mu_{s}^{f}$ is $\sigma$-finite, $\Theta^{f, g}$ extends to a unique measure on $[0, \infty)$ satisfying the equation (4.4) with the stated $\sigma$-finiteness property.

We finally prove the relation (4.5). By letting $t \rightarrow \infty$ in (4.3), we get from (3.2) the identity

$$
\Theta^{f, g}((s, \infty)):=\left\langle\mu_{s}^{f}, \mathbf{H} g\right\rangle, \quad 0<s .
$$


Without loss of generality, we may assume that $\Theta^{f, g}(d u)$ is $\sigma$-finite on $[0, \infty)$. Then by the Fubini theorem and (3.7),

$$
\begin{aligned}
\int_{0}^{\infty} & \left(1-e^{-\alpha u}\right) \Theta^{f, g}(d u)=\alpha \int_{0}^{\infty}\left(\int_{0}^{u} e^{-\alpha s} d s\right) \Theta^{f, g}(d u) \\
& =\alpha \int_{0}^{\infty} e^{-\alpha s}\left(\int_{s+}^{\infty} \Theta^{f, g}(d u)\right) d s=\alpha \int_{0}^{\infty} e^{-\alpha s} \Theta^{f, g}(s, \infty) d s \\
& =\alpha \int_{0}^{\infty} e^{-\alpha s}\left\langle\mu_{s}^{f}, \mathbf{H} g\right\rangle d s=\alpha\left\langle\widehat{\mathbf{H}}_{\alpha} f, \mathbf{H} g\right\rangle=U_{\alpha}(f, g) .
\end{aligned}
$$

\section{Lévy system of time change process and Feller measures}

We continue to work with Borel standard processes $X=\left(X_{t}, \zeta, \mathbf{P}_{x}\right)$ and $\widehat{X}=\left(\widehat{X}_{t}, \widehat{\zeta}, \widehat{\mathbf{P}}_{x}\right)$ on $E$ in weak duality with respect to $m$ satisfying condition (A.1) and a set $F \in \mathcal{B}^{n}$ satisfying the conditions (A.2) and (A.3) formulated in $\S 3$.

Recall the family $S$ of all smooth measures on $E$ for $X$ introduced in $\S 2.3$. Let

$$
S_{F}=\{\mu \in S: \text { the quasi support of } \mu=F \text { q.e. }\} .
$$

$S_{F}$ is non-empty by Corollary 2.4. We take and fix a $\mu \in S_{F}$. There exists a PCAF $A_{t}$ of $X$ with Revuz measure $\mu$ by virtue of Proposition 2.7. The support of $A_{t}$ coincides with $F$ q.e. on account of Proposition 2.6.

In the same way as in the proof of [17, Lemma 5.1.11], one can then show that

$$
\mathbf{P}_{x}\left(\sigma_{F}=\inf \left\{t>0: A_{t}>0\right\}\right)=1, \quad \text { q.e. } x \in E .
$$

Hence, by restricting ourselves to elements outside a certain properly exceptional set including that for $A$, we can assume from the beginning that not only properties (I), (II) and (III) of $\S 3$ hold, but also the following property:

(IV) $A$ is a PCAF of $X$ in the strict sense and

$$
\mathbf{P}_{x}\left(\sigma_{F}=\inf \left\{t>0: A_{t}>0\right\}\right)=1 \quad \text { for every } x \in E .
$$

Note that (I) and (IV) imply that $F$ is just the support of $A$.

We consider the right continuous inverse $\tau_{t}$ of $A$ defined by

$$
\tau_{t}=\inf \left\{s \geq 0: A_{s}>t\right\} \quad \text { with } \inf \emptyset:=\infty,
$$

and the time changed process $Y=\left(Y_{t}, \check{\zeta}, \mathbf{P}_{x}\right)_{x \in F}$ defined by

$$
Y_{t}= \begin{cases}X_{\tau_{t}} & \text { for } 0 \leq t<\check{\zeta}:=A_{\infty} ; \\ \partial & \text { for } t \geq \check{\zeta}\end{cases}
$$

It is known (cf. $[36,(65.9)])$ that $Y$ is a right process with state space $F$. We denote $F \cup\{\partial\}$ by $F_{\partial}$. 
Consider again the random set defined by $(2.7)$ :

$$
M(\omega)=\left\{t \in[0, \zeta(\omega)): X_{t}(\omega) \in F \text { or } X_{t-}(\omega) \in F\right\},
$$

which is relatively closed in $[0, \zeta(\omega))$ by (III) in $\S 3$ almost surely. Let $I$ denote the set of left endpoints of those components of the relatively open set $[0, \zeta(\omega)) \backslash M(\omega)$.

We define, for $t>\sigma_{F}$,

$$
L(t):=\sup [0, t] \cap M
$$

and, for $t \geq 0$,

$$
R(t):=\inf (t, \infty) \cap M=\inf \{s>t: s \in M\}
$$

with the convention that $\inf \emptyset=\infty$. When $t>\sigma_{F}$, we call $(L(t), R(t))$ the excursion straddling on $t$. Clearly $t \mapsto R(t)$ is right continuous and increasing. In view of (3.9), we can see that for every $x \in \mathbf{E}, \mathbf{P}_{x^{-}}$a.s.,

$$
R(t)=\sigma_{F} \circ \theta_{t}+t \quad \text { for every } t .
$$

So $\mathbf{P}_{x}$-a.s.,

$$
R(t) \circ \theta_{s}+s=R(t+s) \quad \text { for every } t, s, \geq 0 .
$$

We also note that

(a) for $t \in M, t=R(t-)$, and

(b) for $t>\sigma_{F}, R(t-)<R(t)$ if and only if $t \in I$; and in this case $t=R(t-)=L(t)$.

On account of (IV), for each fixed $t>0$ and $x \in E, \mathbf{P}_{x}$-a.s.,

$$
\tau_{A_{t}}=\inf \left\{s: A_{s}>A_{t}\right\}=\inf \left\{s>t: A_{s-t} \circ \theta_{t}>0\right\}=\sigma_{F} \circ \theta_{t}+t,
$$

and so, by (5.3), $R(t)=\tau_{A_{t}}$. By the right continuity of $t \mapsto \tau_{A_{t}}$ and $t \mapsto R(t)$, we conclude from the above by applying first to rational $t>0$ that for every $x \in E, \mathbf{P}_{x}$-a.s.,

$$
R(t)=\tau_{A_{t}} \quad \text { for every } t \geq 0 .
$$

This means that $t \mapsto A_{t}$ is constant on each connected component of $[0, \zeta(\omega)) \backslash$ $M(\omega)$. Since $A_{t}$ is continuous in $t$ and $Y_{t} \in F, 0 \leq t<\check{\zeta}$, the next lemma follows immediately from (5.4) and property (III).

Lemma 5.1. The following properties hold $\mathbf{P}_{x}$-a.s. for every $x \in E$ :

(i) $Y_{A_{t}}=X_{R(t)}$ for every $t>0$.

(ii) The left limit $Y_{t-}$ exists and in $F$ for any $t \in(0, \check{\zeta})$ and $Y_{A_{t-}}=$ $X_{R(t-)-}$.

We now prove that the time changed process $Y$ is actually $\mu$-special standard. We prepare a lemma.

Lemma 5.2. The right process $Y$ has a right process $\widehat{Y}$ in weak duality with respect to the measure $\mu$. 
Proof. On account of Remark 2.1, the process $\widehat{X}$ also satisfies the condition (A.1). Moreover, by Proposition 2.8, $\mu \in S_{F}$ is also a smooth measure for $\widehat{X}$. Hence a PCAF $\widehat{A}$ of $\widehat{X}$ exists with $\mu$ as Revuz measure by Proposition 2.7. Since the co-quasi support of $\mu$ is q.e. equivalent to $F$ by Corollary 2.5, the support $\widehat{F}$ of $\widehat{A}$ with respect to $\widehat{X}$ equals $F$ q.e. by virtue of Proposition 2.6 applied to $\widehat{X}$. Let $\widehat{Y}$ be the time changed process of $\widehat{X}$ with respect to $\widehat{A}$. $\widehat{Y}$ is a right process whose state space equals $\widehat{F}$. We shall show that $Y$ and $\widehat{Y}$ are in weak duality with respect to the measure $\mu$.

For $\alpha>0$, we introduce the $\alpha$-energy functional of an $\alpha$-co-excessive function $u$ and an $\alpha$-excessive function $v$ with respect to $X$ by

$$
L^{\alpha}(u, v)=\lim _{\beta \rightarrow \infty} \beta\left(u, v-\beta G_{\alpha+\beta} v\right),
$$

which, by the weak duality, also equals

$$
\lim _{\beta \rightarrow \infty} \beta\left(u-\beta \widehat{G}_{\alpha+\beta} u, v\right) .
$$

Recall the $\alpha$-potential operator $U_{A}^{\alpha}$ associated with $A$ defined by (2.12). The $\alpha$-co-potential operator $\widehat{U}_{\widehat{A}}^{\alpha}$ associated with $\widehat{A}$ is defined analogously. Due to the fundamental Revuz formula (2.13) and the equation

$$
U_{A}^{\alpha} g-U_{A}^{\alpha+\beta} g-\beta G_{\alpha+\beta} U_{A}^{\alpha} g=0,
$$

we have

$$
L^{\alpha}\left(\widehat{U}_{\hat{A}}^{\alpha} f, U_{A}^{\alpha} g\right)=\left\langle\widehat{U}_{\hat{A}}^{\alpha} f, g\right\rangle_{\mu} \quad \text { for } f, g \in \mathcal{B}^{+}(E),
$$

where $\langle f, g\rangle_{\mu}$ denotes the integral $\int f(x) g(x) \mu(d x)$. Similarly we see that the left hand side of (5.5) is equal to $\left\langle f, U_{A}^{\alpha} g\right\rangle_{\mu}$ and consequently

$$
\left\langle\widehat{U}_{\hat{A}}^{\alpha} f, g\right\rangle_{\mu}=\left\langle f, U_{A}^{\alpha} g\right\rangle_{\mu} \quad \text { for } f, g \in \mathcal{B}^{+}(F) .
$$

Next we put for $p>0$

$$
U_{p, A}^{\alpha} g(x)=\mathbf{E}_{x}\left[\int_{0}^{\infty} e^{-\alpha t-p A_{t}} g\left(X_{t}\right) d A_{t}\right],
$$

and observe the equation

$$
U_{p, A}^{\alpha} g-U_{A}^{\alpha} g+p U_{A}^{\alpha} U_{p, A}^{\alpha} g=0 .
$$

An analogous quantity and equation can be associated with $\widehat{A}$.

For the moment, we assume that $\mu(F)<\infty$ and $U_{A}^{\alpha} 1, \widehat{U}_{\hat{A}}^{\alpha} 1$ are bounded on $E$. Note that under this assumption, by linearity, identity (5.6) holds for any $f, g \in \mathcal{B}_{b}(F)$. Now taking $f, g \in \mathcal{B}_{b}^{+}(F)$ and replacing $f$ and $g$ in the equation (5.6) by

$$
f-p \widehat{U}_{p, \hat{A}}^{\alpha} f \quad \text { and } \quad g-p U_{p, A}^{\alpha} g
$$


respectively, we arrive at

$$
\left\langle\widehat{U}_{p, \hat{A}}^{\alpha} f, g\right\rangle_{\mu}=\left\langle f, U_{p, A}^{\alpha} g\right\rangle_{\mu} \quad \text { for } f, g \in \mathcal{B}_{b}^{+}(E) .
$$

Now for a general $\mu \in S_{F}$, we can take an associated $X$-nest $\left\{E_{n}\right\}$ satisfying condition (iii) of Proposition 2.8. We then let

$$
\mu_{n}=1_{E_{n}} \cdot \mu, \quad A_{t}^{n}=\int_{0}^{t} 1_{E_{n}}\left(X_{s}\right) d A_{s}, \quad \widehat{A}_{t}^{n}=\int_{0}^{t} 1_{E_{n}}\left(\widehat{X}_{s}\right) d \widehat{A}_{s}
$$

to get the equation (5.7) holding for $\mu_{n}, A^{n}, \widehat{A}^{n}$. Replacing $f, g$ in the resulting equation by $1_{E_{\ell}} \cdot f, 1_{E_{\ell}} \cdot g$, respectively, and letting first $n \rightarrow \infty$ and then $\ell \rightarrow \infty$, we see the validity of (5.7) for general $\mu$. Finally, by letting $\alpha \downarrow 0$, we obtain the duality relation of the $p$-resolvents of $Y$ and $\widehat{Y}$ with respect to $\mu$.

It follows from Lemma 5.2 that the measure $\mu$ is $Y$-excessive. In the sequel, the $\mu$-polar sets and $\mu$-semipolar sets for the process $Y$ will be called $\mu^{Y}$-polar and $\mu^{Y}$-semipolar, respectively, in order to distinguish them from the $m$-polar and $m$-semipolar sets for $X$.

LEMMA 5.3.

(i) A subset $B \subset F$ is m-polar if and only if it is $\mu^{Y}$-polar; a subset $B \subset F$ is $m$-co-polar if and only if it is $\mu^{Y}$-co-polar.

(ii) Every $\mu^{Y}$-semipolar set is $\mu^{Y}$-polar, and every $\mu^{Y}$-co-semipolar set is $\mu^{Y}$-co-polar

(iii) The $\mu^{Y}$-polarity, $\mu^{Y}$-semipolarity, $\mu^{Y}$-co-polarity and $\mu^{Y}$-co-semipolarity are all the same.

Proof. (i) This part in fact has been (implicitly) established in Fitzsimmons [10, Proposition 4]. Although the statement in [10] assumes that $X$ is symmetric, its proof does not rely on the symmetry of $X$ and uses only the property that $X$ is a standard process and the condition that $m$-semipolar is $m$-polar. Fitzsimmons' argument uses Hunt's balayage theorem. For reader's convenience, we present below a slightly different proof, without using Hunt's deep result.

If $B \subset F$ is $m$-polar, by (iv) of $\S 2.1$, it is contained in a Borel properly $X$-exceptional set $N$. Since $E \backslash N$ is $X$-invariant and $Y$ is a time change of $X$ living on $F, F \backslash N$ is $Y$-invariant. Since the smooth measure $\mu$ does not charge on $m$-polar set, we have $\mu(N)=0$. Thus $N \cap F$ is a $\mu^{Y}$-polar set by (iv) of $\S 2.1$, and so is $B$. This shows that any $m$-polar subset of $F$ is a $\mu^{Y}$-polar set.

Conversely, if there were a nearly Borel (of $X$ ) set $B \subset F$ that is $\mu^{Y}$ polar but not $m$-polar, there would exist a compact subset $K$ of $B$ such that $K$ is not $m$-polar by [36, p. 57]. Define $\sigma_{K}:=\inf \left\{t>0: X_{t} \in K\right\}$ and $f(x):=\mathbf{P}_{x}\left(\sigma_{K}<\infty\right)$. Then $f$ is an excessive function of $X$. 
In view of (I) of $\S 3,(5.3)$ and (5.4), we then have, $\mathbf{P}_{x}$-a.s. on $\left\{\sigma_{K}<\infty\right\}$, for $x \in E$,

$$
\sigma_{K}=R\left(\sigma_{K}\right)=\tau_{A_{\sigma_{K}}} \quad \text { and } \quad Y_{A_{\sigma_{K}}}=X_{\sigma_{K}} \in K .
$$

So if we define $\sigma_{K}^{Y}:=\inf \left\{t>0: Y_{t} \in K\right\}$, then

$$
f(x)=\mathbf{P}_{x}\left(\sigma_{K}<\infty\right)=\mathbf{P}_{x}^{Y}\left(\sigma_{K}^{Y}<\infty\right), \quad x \in F .
$$

Here, to distinguish it from $\mathbf{P}_{x}$ for the process $X, \mathbf{P}_{x}^{Y}$ denotes the law of the process $Y$ starting from $x$. Since $K$ is $\mu^{Y}$-polar, $\int_{F} f(x) \mu(d x)=0$. On the other hand, by the Revuz identity (2.11),

$$
\uparrow \lim _{t \downarrow 0} \frac{1}{t} \mathbf{E}_{m}\left[\int_{0}^{t} f\left(X_{t}\right) d A_{t}\right]=\int_{F} f(x) \mu(d x)=0,
$$

and consequently,

$$
0=\mathbf{E}_{m}\left[\int_{0}^{\infty} f\left(X_{t}\right) d A_{t}\right] \geq \mathbf{E}_{m}\left[\Lambda(\omega) ; \sigma_{K}<\infty\right],
$$

where $\Lambda(\omega)$ is the random variable defined by

$$
\Lambda(\omega):=\mathbf{E}_{X_{\sigma_{K}(\omega)}(\omega)}\left[\int_{0}^{\infty} f\left(X_{s}\right) d A_{s}\right] .
$$

Denote by $K^{r}$ the set of all regular points of $K$ for $X$. Then $X_{\sigma_{K}} \in K^{r}$ $\mathbf{P}_{m}$-a.s. on $\left\{\sigma_{K}<\infty\right\}$ because $K \backslash K^{r}$ is semipolar for the process $X$ and hence $m$-polar by (A.1) in $\S 2.2$. Since $f>0$ on $K^{r}$, the set $\{f>0\}$ is finely open, $K^{r} \subset F^{r}=F$ by (I) in $\S 3$ and $F$ is the support of the PCAF $A$ by (IV) in $\S 5$, we see that $\Lambda>0 \mathbf{P}_{m}$-a.s. on $\left\{\sigma_{K}<\infty\right\}$. This contradicts the inequality (5.8) and our assumption that $K$ is not $m$-polar. Thus we have shown that every $\mu^{Y}$-polar subset of $F$ must be $m$-polar.

Applying the above argument to the dual processes $\widehat{X}$ and $\widehat{Y}$, we obtain that every $\mu^{Y}$-co-polar subset of $F$ is $m$-co-polar. This completes the proof of (i).

(ii) Assume that $B \subset F$ is a $\mu^{Y}$-semipolar set for the process $Y$. Then by (vi) of $\S 2.1$,

$$
\mathbf{P}_{y}\left(Y_{t} \in B \text { for uncountably many } t\right)=0
$$

for $Y$-q.e. $y \in F$ and hence by (i) for q.e. $y \in F$. On the other hand, if we define the time sets

$$
R(X):=\left\{t: X_{t} \in B\right\} \quad \text { and } \quad R(Y):=\left\{t: Y_{t} \in B\right\},
$$

then it follows from $[2, \mathrm{~V}(3.8)]$ that for $x \in E, \mathbf{P}_{x}$-a.s.,

$$
R(Y) \subset R(X) \quad \text { and } \quad R(X) \backslash R(Y) \text { is at most countable. }
$$


Therefore, by the strong Markov property of $X$,

$$
\begin{aligned}
\mathbf{P}_{m} & \left(X_{t} \in B \text { for uncountably many } t\right) \\
& =\mathbf{E}_{m}\left[\mathbf{P}_{X_{\sigma_{F}}}\left(X_{t} \in B \text { for uncountably many } t\right)\right] \\
& =\mathbf{E}_{m}\left[\mathbf{P}_{X_{\sigma_{F}}}\left(Y_{t} \in B \text { for uncountably many } t\right)\right]=0 .
\end{aligned}
$$

This implies that $B$ is $m$-semipolar by virtue of (vi) of $\S 2.1$. So $B$ is $m$-polar, and hence, by (i), is $\mu^{Y}$-polar.

Applying the above argument to the dual process $\widehat{Y}$, we see that every $\mu^{Y}$-co-semipolar set is $\mu^{Y}$-co-polar.

(iii) By (ii) of $\S 2.1$, a set $B \subset F$ is $m$-polar if and only if it is $m$-co-polar. Thus we have that, by (i), a subset of $F$ is $\mu^{Y}$-polar if and only if it is $\mu^{Y}$-copolar. This together with (ii) establishes (iii) of this lemma.

Proposition 5.4. The process $Y$ is a $\mu$-special standard process on $F$ having another $\mu$-special standard process $\widehat{Y}$ on $F$ in weak dual with respect to the measure $\mu$. Moreover, the semigroup of $Y$ maps bounded nearly Borel measurable functions (with respect to $Y$ ) on $F$ into bounded nearly Borel measurable functions (with respect to $Y$ ) on $F$, and the semigroup of $\widehat{Y}$ maps bounded nearly Borel measurable functions (with respect to $\widehat{Y}$ ) on F into bounded nearly Borel measurable functions (with respect to $\widehat{Y}$ ) on $F$.

Proof. On account of Remark 2.10, the first assertion follows from Lemma 5.1, Lemma 5.2 and Lemma 5.3(iii). The second assertion follows from the fact that $Y$ and $\widehat{Y}$ are time changes of the Borel standard processes $X$ and $\widehat{X}$, respectively.

Combining Proposition 5.4 with Lemma 2.12, we can conclude that the time changed process $Y$ on $F$ admits a Lévy system $(\check{N}, \check{H})$. That is, $\check{N}(x, d y)$ is a kernel on $\left(F_{\partial}, \mathcal{B}\left(F_{\partial}\right)\right)$ and $\check{H}$ is a PCAF of $Y$ with bounded 1-potential such that for any nonnegative Borel function $f$ on $F \times F_{\partial}$ that vanishes on the diagonal and is extended to be zero elsewhere,

$$
\mathbf{E}_{x}\left[\sum_{s \leq t} f\left(Y_{s-}, Y_{s}\right)\right]=\mathbf{E}_{x}\left[\int_{0}^{t} \int_{F_{\partial} \backslash N} f\left(X_{s}, y\right) \check{N}\left(X_{s}, d y\right) d \check{H}_{s}\right]
$$

for q.e. (or equivalently $\mu^{Y}$-q.e.) $x \in F$ and $t \geq 0$. Here, $\check{\zeta}$ is the lifetime of $Y$ and $Y_{\check{\zeta}-}$ is defined by

$$
Y_{\breve{\zeta}-}:= \begin{cases}\lim _{t \uparrow \check{\zeta}} Y_{t}, & \text { if the limit } \lim _{t \uparrow \check{\zeta}} Y_{t} \text { exists in } F \\ \partial, & \text { otherwise. }\end{cases}
$$


The Revuz measure of $\check{H}$ with respect to the $Y$-excessive measure $\mu$ will be denoted by $\check{\mu}_{\breve{H}}$. Define

$$
\check{J}(d x, d y):=\check{N}(x, d y) \check{\mu}_{\breve{H}}(d x) \quad \text { and } \quad \check{\kappa}:=\check{N}(x,\{\partial\}) \check{\mu}_{\breve{H}}(d x) .
$$

We call $\check{J}$ and $\check{\kappa}$ the jumping measure and the killing measure of $Y$, respectively.

By (5.9), we have then the formula for the jumping and killing measures:

$$
\int_{F \times F \backslash d} \Psi(x, y) \check{J}(d x, d y)=\uparrow \lim _{t \downarrow 0} \frac{1}{t} \mathbf{E}_{\mu}\left[\sum_{0<s \leq t} \Psi\left(Y_{s-}, Y_{s}\right)\right],
$$

for any $\Psi \in \mathcal{B}^{+}(F \times F)$ that vanishes along the diagonal and is extended to zero elsewhere, and any $f \in \mathcal{B}^{+}(F)$ that is extended to be zero off $F$,

$$
\int_{F} f(x) \check{\kappa}(d x)=\uparrow \lim _{t \downarrow 0} \frac{1}{t} \mathbf{E}_{\mu}\left[f\left(Y_{\breve{\zeta}-}\right) ; \check{\zeta} \leq t\right],
$$

We note that, since $\Lambda(\omega, d t)=\Psi\left(Y_{s-}, Y_{s}\right) \epsilon_{s}(d t)$ is a homogeneous random measure for $Y$, the jumping measure $\breve{J}$ is well-defined by (5.12) for any right process $Y$ with left limits up to the lifetime and for any $Y$-excessive measure $\mu$.

The following theorem relates the jumping and killing measures of $Y$ to the excursions for the process of $X$ away from $F$.

TheOREM 5.5. For any $\Psi \in \mathcal{B}^{+}(F \times F)$ vanishing along d and $f \in \mathcal{B}^{+}(F)$,

$$
\int_{F \times F} \Psi(x, y) \check{J}(d x, d y)=\uparrow \lim _{s \downarrow 0} \frac{1}{s} \mathbf{E}_{m}\left[\sum_{t \leq s, t \in M, R(t)<\infty} \Psi\left(X_{t-}, X_{R(t)}\right)\right]
$$

and

$$
\int_{F} f(x) \check{\kappa}(d x)=\uparrow \lim _{s \downarrow 0} \frac{1}{s} \mathbf{E}_{m}\left[\sum_{t \leq s, t \in I \cup\{\zeta\}} 1_{\left\{\sigma_{F}=\infty\right\}} \circ \theta_{t} f\left(X_{t-}\right)\right] .
$$

Proof. We note that for $t \in M, t=R(t-)$ and so (5.14) can be rewritten as

$$
\int_{F \times F} \Psi(x, y) \check{J}(d x, d y)=\uparrow \lim _{s \downarrow 0} \frac{1}{s} \mathbf{E}_{m}\left[\sum_{t \leq s, t \in M, R(t)<\infty} \Psi\left(X_{R(t-)-}, X_{R(t)}\right)\right] .
$$

The proof of this theorem is similar to the proofs of [16, Theorem 5.1] and [4, Theorem 4.2] but we present a proof for completeness. Let $A$ be the PCAF of $X$ with Revuz measure $\mu$. 
We take any $\Psi$ as in the statement of the lemma and extend it to $E_{\partial} \times E_{\partial}$ by setting its value outside $F \times F$ to be zero. From the formula (5.12), we have

$$
\int_{F \times F} \Psi(\xi, \eta) \check{J}(d \xi, d \eta)=\uparrow \lim _{\alpha \uparrow \infty} \alpha \mathbf{E}_{\mu}\left[\sum_{0<t<\infty} e^{-\alpha t} \Psi\left(Y_{t-}, Y_{t}\right)\right]
$$

We now make a change of variable, replacing $t$ with $A_{t}$. By virtue of Lemma 5.1 , we then obtain

$$
\begin{aligned}
\int_{F \times F} \Psi(\xi, \eta) \check{J}(d \xi, d \eta) & =\uparrow \lim _{\alpha \uparrow \infty} \alpha \mathbf{E}_{\mu}\left[\sum_{t \in M, R(t)<\infty} e^{-\alpha A_{t}} \Psi\left(X_{R(t-)-}, X_{R(t)}\right)\right] \\
& =\uparrow \lim _{\alpha \uparrow \infty} \int_{F} \alpha \mathbf{E}^{x}\left[\Sigma_{\alpha}\right] \mu(d x),
\end{aligned}
$$

where

$$
\Sigma_{\alpha}:=\sum_{t \in M, R(t)<\infty} e^{-\alpha A_{t}} \Psi\left(X_{R(t-)-}, X_{R(t)}\right)
$$

It follows from $(2.11)$ and $[36,(32.6)]$ that

$$
\begin{aligned}
\int_{F \times F} \Psi(\xi, \eta) \check{J}(d \xi, d \eta) & =\uparrow \lim _{\alpha \uparrow \infty} \alpha \int_{F} \mathbf{E}_{x}\left[\Sigma_{\alpha}\right] \mu(d x) \\
& =\uparrow \lim _{\alpha \uparrow \infty} \alpha\left(\uparrow \lim _{s \downarrow 0} \frac{1}{s} \mathbf{E}_{m}\left[\int_{0}^{s} \mathbf{E}_{X_{u}}\left[\Sigma_{\alpha}\right] d A_{u}\right]\right) \\
& =\uparrow \lim _{s \downarrow 0} \frac{1}{s}\left(\sup _{\alpha>0} \alpha \mathbf{E}_{m}\left[\int_{0}^{s} \Sigma_{\alpha} \circ \theta_{u} d A_{u}\right]\right) .
\end{aligned}
$$

Now

$$
\begin{aligned}
\alpha \mathbf{E}_{m}\left[\int_{0}^{s} \Sigma_{\alpha} \circ \theta_{u} d A_{u}\right] \\
=\alpha \mathbf{E}_{m}\left[\int_{0}^{s} \sum_{t \in M \circ \theta_{u}, R(t+u)<\infty} e^{-\alpha\left(A_{t+u}-A_{u}\right)} \Psi\left(X_{R(t+u-)-}, X_{R(t+u)}\right) d A_{u}\right] \\
=\alpha \mathbf{E}_{m}\left[\int_{0}^{s} e^{\alpha A_{u}} d A_{u}^{\mu} \sum_{t>u, t \in M, R(t)<\infty} e^{-\alpha A_{t}} \Psi\left(X_{R(t-)-}, X_{R(t)}\right)\right]
\end{aligned}
$$




$$
\begin{aligned}
& =\mathbf{E}_{m}\left[\sum_{t \in M, R(t)<\infty} e^{-\alpha A_{t}} \Psi\left(X_{R(t-)-}, X_{R(t)}\right) \int_{0}^{s} 1_{\{t>u\}} d e^{\alpha A_{u}}\right] \\
& =\mathbf{E}_{m}\left[\sum_{t \in M, R(t)<\infty} e^{-\alpha A_{t}} \Psi\left(X_{R(t-)-}, X_{R(t)}\right) \cdot\left(e^{\alpha A_{s \wedge t}}-1\right)\right] \\
& =I_{\alpha, s}^{-}+I_{\alpha, s}^{+},
\end{aligned}
$$

where

$$
\begin{aligned}
& I_{\alpha, s}^{-}=\mathbf{E}_{m}\left[\sum_{t \leq s, t \in M, R(t)<\infty}\left(1-e^{-\alpha A_{t}}\right) \Psi\left(X_{R(t-)-}, X_{R(t)}\right)\right], \\
& I_{\alpha, s}^{+}=\mathbf{E}_{m}\left[\left(e^{\alpha A_{s}}-1\right) \sum_{t>s, t \in M, R(t)<\infty} e^{-\alpha A_{t}} \Psi\left(X_{R(t-)-}, X_{R(t)}\right)\right] .
\end{aligned}
$$

It follows from (5.16) that

$$
\int_{F \times F} \Psi(\xi, \eta) \check{J}(d \xi, d \eta)=\lim _{s \downarrow 0} \frac{1}{s}\left[\sup _{\alpha}\left(I_{\alpha, s}^{-}+I_{\alpha, s}^{+}\right)\right] .
$$

Note that, since $\Psi$ vanishes along the diagonal $d$, we can insert in the summand of $I_{\alpha, s}^{-}$the condition that $\sigma_{F}<t$, which is equivalent to $A_{t}>0$ in view of the property (IV) in $\S 5$.

From (5.17), we can then conclude that

$$
\int_{F \times F} \Psi(\xi, \eta) \check{J}(d \xi, d \eta) \geq \uparrow \lim _{s \downarrow 0} \frac{1}{s} \mathbf{E}_{m}\left[\sum_{t \leq s, t \in M, R(t)<\infty} \Psi\left(X_{R(t-)-}, X_{R(t)}\right)\right],
$$

because we see that $I_{\alpha, s}^{-}$increases to the right hand side of (5.18) as $\alpha \rightarrow \infty$ by taking the above note into account. If the right hand side of (5.18) is infinite, then the desired equality holds true trivially.

Without loss of generality, we may and do assume that the right hand side of (5.18) is finite. In order to obtain the converse inequality to (5.18), observe that we may take a strictly positive $m$-integrable function $f$ on $E$ because $m$ is $\sigma$-finite. By weak duality $G_{1} f$ is then strictly positive on $E$ and $m$-integrable. Define for $n \geq 1$,

$E_{n}=\left\{x \in E: G_{1} f(x)>\frac{1}{n}\right\}, m_{n}=\left.m\right|_{E_{n}}$, and $\tau_{n}=\inf \left\{t \geq 0: X_{t} \notin E_{n}\right\}$.

Then $m_{n}\left(E_{n}\right)<\infty$ and $\lim _{n \rightarrow \infty} \tau_{n}=\zeta, \mathbf{P}_{x^{-}}$a.s. for every $x \in E$. 
Let $X^{n}:=\left(X_{t}^{n}, \tau_{n}, \mathbf{P}_{x}\right)_{x \in E_{n}}$ be the subprocess of $X$ killed upon leaving $E_{n}$; that is,

$$
X_{t}^{n}= \begin{cases}X_{t}, & \text { for } t<\tau_{n} \\ \partial, & \text { for } t \geq \tau_{n}\end{cases}
$$

Since $X^{n}$ is in weak duality under the measure $m_{n}$ with the subprocess of $\widehat{X}$ killed upon leaving $E_{n}$ (see $\S 2$ ), $m_{n}$ is $X^{n}$-excessive. We then define, for any $u \geq 0$ and $n \geq 1$,

$$
\begin{aligned}
\Sigma_{\alpha, u}^{n} & :=\sum_{u<t<\infty, t \in M, R(t)<\tau_{n}} e^{-\alpha A_{t}} \Psi\left(X_{R(t-)-}^{n}, X_{R(t)}^{n}\right) \\
& =\sum_{u<t<\infty, t \in M, R(t)<\tau_{n}} e^{-\alpha A_{t}} \Psi\left(X_{R(t-)-}, X_{R(t)}\right) .
\end{aligned}
$$

Note that $\mathbf{E}_{m_{n}}\left[\left(e^{\alpha A_{s}}-1\right) \sum_{\alpha, s}^{n}\right]$ increases to $I_{\alpha, s}^{+}$as $n \rightarrow \infty$. It can be easily verified that

$$
e^{\alpha A_{s}} \cdot \Sigma_{\alpha, s}^{n}=\Sigma_{\alpha, 0}^{n} \circ \theta_{s}^{n},
$$

where $\theta_{s}^{n}$ is the shift operator for the process $X^{n}: X_{t}^{n} \circ \theta_{s}^{n}=X_{s+t}^{n}$.

We next take a truncation function $\chi_{N}(x)=x \wedge N, x \in \mathbb{R}$ and set

$$
I_{\alpha, s, n, N}^{+}=\mathbf{E}_{m_{n}}\left[\chi_{N}\left(e^{\alpha A_{s}} \cdot \Sigma_{\alpha, s}^{n}\right)-\chi_{N}\left(\Sigma_{\alpha, s}^{n}\right)\right] .
$$

Since $0 \leq \chi_{N}(b)-\chi_{N}(a) \uparrow b-a, N \uparrow \infty$ for $a<b$, we see that $I_{\alpha, s, n, N}^{+}$ increases to $I_{\alpha, s}^{+}$when we let $N \uparrow \infty$ and then $n \uparrow \infty$.

Since the measure $m_{n}$ is $X^{n}$-excessive and finite, we have

$$
\begin{aligned}
I_{\alpha, s, n, N}^{+} & =\mathbf{E}_{m_{n}}\left[\mathbf{E}_{X_{s}^{n}}\left[\chi_{N}\left(\sum_{\alpha, 0}^{n}\right)\right]\right]-\mathbf{E}_{m_{n}}\left[\chi_{N}\left(\Sigma_{\alpha, s}^{n}\right)\right] \\
& \leq \mathbf{E}_{m_{n}}\left[\chi_{N}\left(\Sigma_{\alpha, 0}^{n}\right)\right]-\mathbf{E}_{m_{n}}\left[\chi_{N}\left(\Sigma_{\alpha, s}^{n}\right)\right] \\
& =\mathbf{E}_{m_{n}}\left[\chi_{N}\left(\Sigma_{\alpha, 0}^{n}\right)-\chi_{N}\left(\Sigma_{\alpha, s}^{n}\right)\right] \\
& \leq \mathbf{E}_{m_{n}}\left[\Sigma_{\alpha, 0}^{n}-\Sigma_{\alpha, s}^{n}\right] \\
& \leq \mathbf{E}_{m}\left[\sum_{0<t \leq s, t \in M, R(t)<\infty} e^{-\alpha A_{s}} \Psi\left(X_{R(t-)-}, X_{R(t)}\right)\right] .
\end{aligned}
$$

The last expectation in the above display is finite under the present assumption, since

$$
I_{\alpha, s}^{-}+I_{\alpha, s, n, N}^{+} \leq \mathbf{E}_{m}\left[\sum_{0<t \leq s, t \in M, R(t)<\infty} \Psi\left(X_{R(t-)-}, X_{R(t)}\right)\right] .
$$


Therefore we have from (5.17)

$$
\begin{aligned}
\int_{F \times F} \Psi(\xi, \eta) \check{J}(d \xi, d \eta) & =\lim _{s \downarrow 0} \frac{1}{s} \sup _{\alpha}\left(I_{\alpha, s}^{-}+\lim _{n \rightarrow \infty} \lim _{N \rightarrow \infty} I_{\alpha, s, n, N}^{+}\right) \\
& \leq \lim _{s \downarrow 0} \frac{1}{s} \mathbf{E}_{m}\left[\sum_{0<t \leq s, t \in M, R(t)<\infty} \Psi\left(X_{R(t-)-}, X_{R(t)}\right)\right],
\end{aligned}
$$

which, together with (5.18), completes the proof for (5.14).

Next we show that for any $f \in \mathcal{B}^{+}(F)$,

$$
\int_{F} f(x) \check{\kappa}(d x)=\uparrow \lim _{s \downarrow 0} \frac{1}{s} \mathbf{E}_{m}\left[\sum_{t \leq s, t \in M \cup\{\zeta\}} 1_{\left\{\sigma_{F}=\infty\right\}} \circ \theta_{t} f\left(X_{t-}\right)\right] .
$$

Since the lifetime $\check{\zeta}$ of $Y$ is $A_{\infty}, \tau_{A_{\infty}}=\infty$ and $\tau_{A_{\infty}-}=\eta=\inf \left\{t: A_{t}=\right.$ $\left.A_{\infty}\right\}=\sup \left\{t: X_{t} \in F\right\}$, we have

$$
Y_{\check{\zeta}-}=X_{\tau_{A_{\infty}-}-}=X_{\eta-},
$$

and we have by (5.13) and the Revuz identity (2.11),

$$
\begin{aligned}
\int_{F} f(x) \check{\kappa}(d x) & =\uparrow \lim _{t \downarrow 0} \frac{1}{t} \mathbf{E}_{\mu}\left[f\left(Y_{\check{\zeta}-}\right) ; \check{\zeta} \leq t\right] \\
& =\uparrow \lim _{\alpha \uparrow \infty} \alpha \mathbf{E}_{\mu}\left[e^{-\alpha \check{\zeta}} f\left(Y_{\check{\zeta}-}\right)\right]=\uparrow \lim _{\alpha \uparrow \infty} \alpha \mathbf{E}_{\mu}\left[e^{-\alpha A_{\infty}} f\left(X_{\eta-}\right)\right] \\
& =\uparrow \lim _{\alpha \uparrow \infty} \alpha\left(\uparrow \lim _{s \downarrow 0} \frac{1}{s} \mathbf{E}_{m}\left[\int_{0}^{s}\left(e^{-\alpha A_{\infty}} f\left(X_{\eta-}\right)\right) \circ \theta_{u} d A_{u}\right]\right) \\
& =\uparrow \lim _{s \downarrow 0} \frac{1}{s}\left(\uparrow \lim _{\alpha \uparrow \infty} \alpha \mathbf{E}_{m}\left[\int_{0}^{s \wedge \eta} e^{\alpha A_{u}} e^{-\alpha A_{\infty}} f\left(X_{\eta-}\right) d A_{u}\right]\right) \\
& =\uparrow \lim _{s \downarrow 0} \frac{1}{s}\left(\uparrow \lim _{\alpha \uparrow \infty} \mathbf{E}_{m}\left[\left(e^{\alpha A_{s \wedge \eta}}-1\right) e^{-\alpha A_{\infty}} f\left(X_{\eta-}\right)\right]\right) \\
& =\uparrow \lim _{s \downarrow 0} \frac{1}{s} \mathbf{E}_{m}\left[f\left(X_{\eta-}\right) ; A_{s \wedge \eta}=A_{\infty}\right] \\
& =\uparrow \lim _{s \downarrow 0} \frac{1}{s} \mathbf{E}_{m}\left[f\left(X_{\eta-}\right) ; \eta \leq s\right]=I+I I,
\end{aligned}
$$

where

$$
I=\uparrow \lim _{s \downarrow 0} \frac{1}{s} \mathbf{E}_{m}\left[f\left(X_{\eta-}\right) ; \eta<\zeta, \eta \leq s\right]
$$

and

$$
I I=\uparrow \lim _{s \downarrow 0} \frac{1}{s} \mathbf{E}_{m}\left[f\left(X_{\eta-}\right) ; \eta=\zeta \leq s\right] .
$$

Now

$$
I=\uparrow \lim _{s \downarrow 0} \frac{1}{s} \mathbf{E}_{m}\left[\sum_{t \leq s, t \in M} 1_{\left\{\zeta>0, \sigma_{F}=\infty\right\}} \circ \theta_{t} f\left(X_{t-}\right)\right],
$$


while

$$
I I=\uparrow \lim _{s \downarrow 0} \frac{1}{s} \mathbf{E}_{m}\left[1_{\left\{X_{\zeta-} \in F\right\}} f\left(X_{\zeta-}\right) ; \eta=\zeta \leq s\right] .
$$

On the other hand, for every $s>0$, by $(2.20)$

$$
\mathbf{E}_{m}\left[1_{\left\{X_{\zeta-} \in F\right\}} f\left(X_{\zeta-}\right) ; \eta<\zeta \leq s\right]=0 .
$$

Hence

$$
I I=\uparrow \lim _{s \downarrow 0} \frac{1}{s} \mathbf{E}_{m}\left[1_{\left\{X_{\zeta-} \in F\right\}} f\left(X_{\zeta-}\right) ; \zeta \leq s\right] .
$$

This combined with the expression for I proves (5.19).

Note that for $t \in M \backslash I$ with $\sigma_{F} \circ \theta_{t}=\infty$, we have $t=R(t-)=R(t)=\infty$ and so (5.15) follows.

In $\S 3$, we have considered the Lévy system $(N, H)$ of $X$ and the Revuz measure $\mu_{H}$ of $H$. We define the jumping measure $J$ and the killing measure $\kappa$ of $X$ by

$$
J(d x, d y):=\mu_{H}(d x) N(x, d y) \quad \text { and } \quad \kappa(d x):=N(x,\{\partial\}) \mu_{H}(d x),
$$

respectively. Combining Corollary 3.5 with Theorem 5.5, we can establish the following identification of the jumping measure $\breve{J}$ and the killing measure $\breve{\kappa}$ of the time changed process $Y$.

TheOREM 5.6. We have

$$
\check{J}=U+\left.J\right|_{F \times F},
$$

and

$$
\check{\kappa}(d x)=V(d x)+\left.\kappa(d x)\right|_{F},
$$

where $U$ and $V$ are the Feller measure and supplement Feller measure defined by (3.3) and (3.4), respectively.

Proof. The sum in the right hand side of the identity in Theorem 5.5 can be divided into two parts: $t \in I$ where $t=R(t-)<R(t)=t+\sigma_{F} \circ \theta_{t}$, and $t \in M \backslash I$ where $t=R(t-)=R(t)$. Thus for $\Psi \geq 0$ on $F \times F$ vanishing along 
the diagonal $d$ and off $F \times F$,

$$
\begin{aligned}
& \lim _{s \downarrow 0} \frac{1}{s} \mathbf{E}_{m} {\left[\sum_{t \leq s, t \in M, R(t)<\infty} \Psi\left(X_{R(t-)-}, X_{R(t)}\right)\right] } \\
&=\lim _{s \downarrow 0} \frac{1}{s} \mathbf{E}_{m}\left[\sum_{t \leq s, t \in I, R(t)<\infty} \Psi\left(X_{t-}, X_{\sigma_{F}} \circ \theta_{t}\right)\right] \\
& \quad+\lim _{s \downarrow 0} \frac{1}{s} \mathbf{E}_{m}\left[\sum_{t \leq s, X_{t-}, X_{t} \in F} \Psi\left(X_{t-}, X_{t}\right) .\right] \\
&=\int_{F \times F} \Psi(x, y) U(d x, d y)+\int_{F \times F} \Psi(x, y) J(d x, d y) .
\end{aligned}
$$

In the last equality, (3.19) and the Lévy system for $X$ are used. This proves (5.21).

By (3.20) and the Lévy system for $X$, for any $f \geq 0$ that vanishes on $E_{\partial} \backslash F$,

$$
\begin{aligned}
& \lim _{s \downarrow 0} \frac{1}{s} \mathbf{E}_{m} {\left[\sum_{t \leq s, t \in I \cup\{\zeta\}} 1_{\left\{\sigma_{F}=\infty\right\}} \circ \theta_{t} f\left(X_{t-}\right)\right] } \\
&=\lim _{s \downarrow 0} \frac{1}{s} \mathbf{E}_{m}\left[\sum_{t \leq s, t \in I} f\left(X_{t-}\right) 1_{\left\{\zeta>0, \sigma_{F}=\infty\right\}} \circ \theta_{t}\right] \\
& \quad+\lim _{s \downarrow 0} \frac{1}{s} \mathbf{E}_{m}\left[1_{\left\{X_{\zeta-} \in F\right\}} f\left(X_{\zeta-}\right) ; \zeta \leq s\right] . \\
&=\int_{F} f(x) V(d x)+\int_{F} f(x) \kappa(d x) .
\end{aligned}
$$

In view of (5.15), this establishes the identity $\check{\kappa}(d x)=V(d x)+\left.\kappa(d x)\right|_{F}$.

This theorem extends a recent result of the authors [4] from symmetric Markov processes $X$ to non-symmetric Markov processes having a weak dual. It in particular shows that the jumping and killing measures of $Y$ are independent of the choice of $\mu \in S_{F}$. In fact, this independence of $\mu \in S_{F}$ can also be deduced from Theorem 5.5. One can also apply results from [11] and [19] to see this independence at the sample path level; it is shown in [19] that two PCAF's having the same fine support are time changes of each other by a strictly increasing PCAF, while [11, Theorem 6.2] shows that the correspondence between PCAF and its Revuz measure is invariant under time change.

From the jumping measure $\breve{J}$ and $\check{\kappa}$, one can easily deduce a Lévy system for $Y$. 


\section{REFERENCES}

[1] R. M. Blumenthal, Excursions of Markov processes, Probability and its Applications, Birkhäuser Boston Inc., Boston, MA, 1992. MR 1138461 (93b:60159)

[2] R. M. Blumenthal and R. K. Getoor, Markov processes and potential theory, Pure and Applied Mathematics, Vol. 29, Academic Press, New York, 1968. MR 0264757 (41 \#9348)

[3] A. Benveniste and J. Jacod, Systèmes de Lévy des processus de Markov, Invent. Math. 21 (1973), 183-198. MR 0343375 (49 \#8117)

[4] Z.-Q. Chen, M. Fukushima, and J. Ying, Traces of symmetric Markov processes and their characterizations, Ann. Probab. 34 (2006), 1052-1102.

[5] J. L. Doob, Boundary properties for functions with finite Dirichlet integrals, Ann. Inst. Fourier (Grenoble) 12 (1962), 573-621. MR 0173783 (30 \#3992)

[6] W. Feller, On boundaries and lateral conditions for the Kolmogorov differential equations, Ann. of Math. (2) 65 (1957), 527-570. MR 0090928 (19,892b)

[7] P. J. Fitzsimmons, On two results in the potential theory of excessive measures, Seminar on stochastic processes, 1986 (Charlottesville, Va., 1986), Progr. Probab. Statist., vol. 13, Birkhäuser Boston, Boston, MA, 1987, pp. 21-29. MR 902424 (89b:60177)

[8] - On the excursions of Markov processes in classical duality, Probab. Theory Related Fields 75 (1987), 159-178. MR 885460 (88g:60174)

[9] _ Markov processes and nonsymmetric Dirichlet forms without regularity, J. Funct. Anal. 85 (1989), 287-306. MR 1012207 (90i:60053)

[10] - Time changes of symmetric Markov processes and a Feynman-Kac formula, J. Theoret. Probab. 2 (1989), 487-501. MR 1011201 (91h:60076)

[11] P. J. Fitzsimmons and R. K. Getoor, Revuz measures and time changes, Math. Z. 199 (1988), 233-256. MR 958650 (89h:60124)

[12] _ Smooth measures and continuous additive functionals of right Markov processes, Itô's stochastic calculus and probability theory, Springer, Tokyo, 1996, pp. 3149. MR 1439516 (98g:60137)

[13] _ Excursion theory revisited, Illinois J. Math. 50 (2006).

[14] M. Fukushima, On boundary conditions for multi-dimensional Brownian motions with symmetric resolvent densities, J. Math. Soc. Japan 21 (1969), 58-93. MR 0236998 (38 \#5291)

[15] Almost polar sets and an ergodic theorem, J. Math. Soc. Japan 26 (1974), 17-32. MR 0350871 (50 \#3363)

[16] M. Fukushima, P. He, and J. Ying, Time changes of symmetric diffusions and Feller measures, Ann. Probab. 32 (2004), 3138-3166. MR 2094441 (2005j:60143)

[17] M. Fukushima, Y. Ōshima, and M. Takeda, Dirichlet forms and symmetric Markov processes, de Gruyter Studies in Mathematics, vol. 19, Walter de Gruyter \& Co., Berlin, 1994. MR 1303354 (96f:60126)

[18] M. Fukushima and H. Tanaka, Poisson point processes attached to symmetric diffusions, Ann. Inst. H. Poincaré Probab. Statist. 41 (2005), 419-459. MR 2139028 (2006d:60125)

[19] R. K. Getoor, Some remarks on continuous additive functionals, Ann. Math. Statist 38 (1967), 1655-1660. MR 0216573 (35 \#7404)

[20] _ Markov processes: Ray processes and right processes, Lecture Notes in Mathematics, vol. 440, Springer-Verlag, Berlin, 1975. MR 0405598 (53 \#9390)

[21] _ Excursions of a Markov process, Ann. Probab. 7 (1979), 244-266. MR 525052 (80j:60103)

[22] _ Excessive measures, Probability and its Applications, Birkhäuser Boston Inc., Boston, MA, 1990. MR 1093669 (92i:60135) 
[23] R. K. Getoor and M. J. Sharpe, Excursions of dual processes, Adv. in Math. 45 (1982), 259-309. MR 673804 (84h:60129)

[24] _ Naturality, standardness, and weak duality for Markov processes, Z. Wahrsch. Verw. Gebiete 67 (1984), 1-62. MR 756804 (86f:60093)

[25] P. Hsu, On excursions of reflecting Brownian motion, Trans. Amer. Math. Soc. 296 (1986), 239-264. MR 837810 (87k:60182)

[26] P. A. Jacobs, Excursions of a Markov process induced by continuous additive functionals, Z. Wahrsch. Verw. Gebiete 44 (1978), 325-336. MR 509205 (80a:60099)

[27] H. Kaspi, Excursion laws of Markov processes in classical duality, Ann. Probab. 13 (1985), 492-518. MR 781419 (86h:60139)

[28] H. Kunita, General boundary conditions for multi-dimensional diffusion processes., J. Math. Kyoto Univ. 10 (1970), 273-335. MR 0270445 (42 \#5333)

[29] Y. Le Jan, Balayage et formes de Dirichlet, Z. Wahrsch. Verw. Gebiete 37 (1976/77), 297-319. MR 571671 (81k:60082)

[30] _ Mesures associées à une forme de Dirichlet. Applications, Bull. Soc. Math. France 106 (1978), 61-112. MR 508949 (81c:31014)

[31] B. Maisonneuve, Exit systems, Ann. Probability 3 (1975), 399-411. MR 0400417 (53 \#4251)

[32] J. B. Mitro, Dual Markov processes: construction of a useful auxiliary process, Z. Wahrsch. Verw. Gebiete 47 (1979), 139-156. MR 523166 (80g:60075)

[33] M. Motoo, Application of additive functionals to the boundary problem of Markov processes. Lévy's system of U-processes, Proc. Fifth Berkeley Sympos. Math. Statist. and Probability (Berkeley, Calif., 1965/66), Vol. II: Contributions to Probability Theory, Part 2, Univ. California Press, Berkeley, Calif., 1967, pp. 75-110. MR 0220349 (36 \#3414)

[34] J. Neveu, Une generalisation des processus à accroissements positifs independants, Abh. Math. Sem. Univ. Hamburg 25 (1961), 36-61. MR 0130714 (24 \#A574)

[35] D. Revuz, Mesures associées aux fonctionnelles additives de Markov. I, Trans. Amer. Math. Soc. 148 (1970), 501-531. MR 0279890 (43 \#5611)

[36] M. Sharpe, General theory of Markov processes, Pure and Applied Mathematics, vol. 133, Academic Press Inc., Boston, MA, 1988. MR 958914 (89m:60169)

[37] M. L. Silverstein, Symmetric Markov processes, Springer-Verlag, Berlin, 1974. MR 0386032 (52 \#6891)

[38] — The sector condition implies that semipolar sets are quasi-polar, Z. Wahrscheinlichkeitstheorie und Verw. Gebiete 41 (1977/78), 13-33. MR 0467934 (57 \#7784)

[39] S. Watanabe, On discontinuous additive functionals and Lévy measures of a Markov process, Japan. J. Math. 34 (1964), 53-70. MR 0185675 (32 \#3137)

[40] M. Weil, Propriétés de continuité fine des fonctions coexcessives, Z. Wahrscheinlichkeitstheorie und Verw. Gebiete 12 (1969), 75-86. MR 0256466 (41 \#1122)

Zhen-Qing Chen, Department of Mathematics, University of Washington, SeatTLE, WA 98195, USA

E-mail address: zchen@math.washington.edu

Masatoshi Fukushima, Department of Mathematics, Kansai University, Suita, OSAKA 564-8680, JAPAN

E-mail address: fuku2@mx5.canvas.ne.jp

Jiangang Ying, Department of Mathematics, Fudan University, Shanghai, China

E-mail address: jgying@fudan.edu.cn 